\author{
SANDIA REPORT \\ SAND2009-6399 \\ Unlimited Release \\ Printed October 2009
}

\title{
Effects of Composition on the Mechanical Response of Alumina-Filled Epoxy
}

\author{
Stephen T. Montgomery
}

Prepared by

Sandia National Laboratories

Albuquerque, New Mexico 87185 and Livermore, California 94550

Sandia is a multiprogram laboratory operated by Sandia Corporation, a Lockheed Martin Company, for the United States Department of Energy's

National Nuclear Security Administration under Contract DE-AC04-94AL85000.

Approved for public release; further dissemination unlimited.

Sandia National Laboratories 
Issued by Sandia National Laboratories, operated for the United States Department of Energy by Sandia Corporation.

NOTICE: This report was prepared as an account of work sponsored by an agency of the United States Government. Neither the United States Government, nor any agency thereof, nor any of their employees, nor any of their contractors, subcontractors, or their employees, make any warranty, express or implied, or assume any legal liability or responsibility for the accuracy, completeness, or usefulness of any information, apparatus, product, or process disclosed, or represent that its use would not infringe privately owned rights. Reference herein to any specific commercial product, process, or service by trade name, trademark, manufacturer, or otherwise, does not necessarily constitute or imply its endorsement, recommendation, or favoring by the United States Government, any agency thereof, or any of their contractors or subcontractors. The views and opinions expressed herein do not necessarily state or reflect those of the United States Government, any agency thereof, or any of their contractors.

Printed in the United States of America. This report has been reproduced directly from the best available copy.

Available to DOE and DOE contractors from

U.S. Department of Energy

Office of Scientific and Technical Information

P.O. Box 62

Oak Ridge, TN 37831

Telephone: $\quad$ (865) 576-8401

Facsimile: (865) 576-5728

E-Mail: $\quad$ reports@adonis.osti.gov

Online ordering: http://www.osti.gov/bridge

Available to the public from

U.S. Department of Commerce

National Technical Information Service

5285 Port Royal Rd.

Springfield, VA 22161

Telephone: $\quad$ (800) 553-6847

Facsimile: (703) 605-6900

E-Mail: $\quad$ orders@ntis.fedworld.gov

Online order: $\quad$ http://www.ntis.gov/help/ordermethods.asp?loc=7-4-0\#online

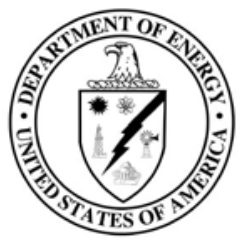


SAND2009-6399

Unlimited Release

Printed October 2009

\title{
Effects of Composition on the Mechanical Response of Alumina-Filled Epoxy
}

\author{
Stephen T. Montgomery \\ Solid Mechanics Department \\ Sandia National Laboratories \\ P.O. Box 5800 \\ Albuquerque, NM 87185-0372
}

\begin{abstract}
The effect of composition on the elastic responses of alumina particle-filled epoxy composites is examined using isotropic elastic response models relating the average stresses and strains in a discretely reinforced composite material consisting of perfectly bonded and uniformly distributed particles in a solid isotropic elastic matrix. Responses for small elastic deformations and large hydrostatic and plane-strain compressions are considered. The response model for small elastic deformations depends on known elastic properties of the matrix and particles, the volume fraction of the particles, and two additional material properties that reflect the composition and microstructure of the composite material. These two material properties, called strain concentration coefficients, are characterized for eleven alumina-filled epoxy composites. It is found that while the strain concentration coefficients depend strongly on the volume fraction of alumina particles, no significant dependence on particle morphology and size is observed for the compositions examined. Additionally, an analysis of the strain concentration coefficients reveals a remarkably simple dependency on the alumina volume fraction. Responses for large hydrostatic and plane-strain compressions are obtained by generalizing the equations developed for small deformation, and letting the alumina volume fraction in the composite increase with compression. The large compression plane-strain response model is shown to predict equilibrium Hugoniot states in alumina-filled epoxy compositions remarkably well.
\end{abstract}




\section{Acknowledgements}

The author would like to thank M. L. Hilborn for fabricating the ALOX materials examined in this study and D. R. Bronowski for density and ultrasonic wave speed measurements on the $20 \%$ AA5 alumina-filled epoxy samples. The author would also like to recognize useful discussions with R. E. Setchell and M. U. Anderson on the compositional dependence of the equilibrium shock-wave stress intensity in alumina-filled epoxy composites and thank T. W. Scofield for his support to ensure the results of this study were documented. 


\section{Contents}

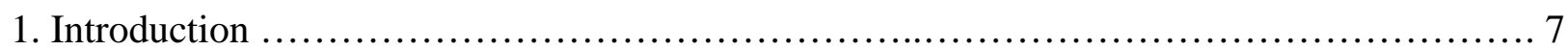

2. A Model for the Elastic Response for Discretely Reinforced Composites ..................... 9

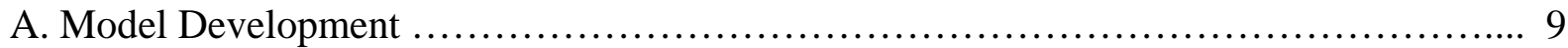

B. Parameter Characterization ................................................................. 12

C. Strain Concentration Coefficient Estimates .......................................... 14

3. Characterization of Several Alumina-Filled Epoxy Compositions ….................... 17

A. Average Elastic Properties of the Epoxy Matrix and Alumina Reinforcement ............... 19

B. Strain Concentration Coefficients for T64 Compositions ................................ 21

C. Strain Concentration Coefficients for AA18 Compositions ............................ 24

D. Strain Concentration Coefficients for AA5 Compositions ............................... 25

E. Effect of Particle Type on Strain Concentration Coefficients ............................. 26

F. An Examination of the Analytic Approximations ......................................... 26

4. The Response of Alumina-Filled Epoxy to Large Compression ............................. 31

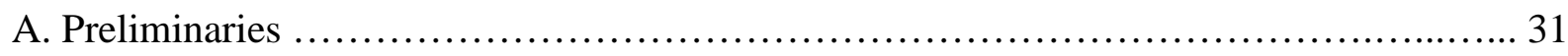

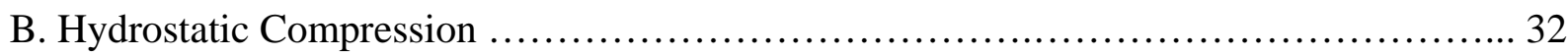

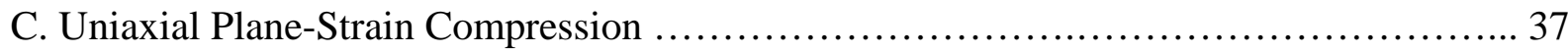

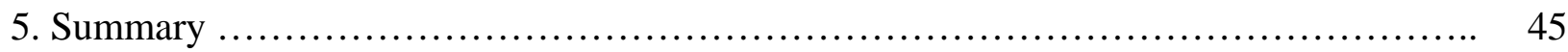

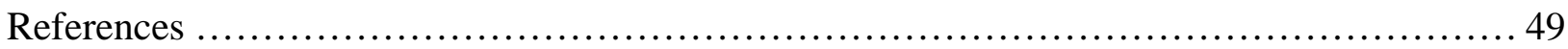

\section{Figures}

Fig. 1 Microscope images of T64 and AA18 alumina particles above the corresponding images of polished surfaces of compositions, containing roughly $43 \%$ alumina by volume, fabricated from these two particle types

Fig. 2 Values of $\kappa_{1}$ and $\gamma_{1}$ versus alumina volume fraction calculated from measured acoustic speeds and density for T64 compositions ............................ 22

Fig. 3 Values of $1 / \kappa_{1}$ and $1 / \gamma_{1}$ versus alumina volume fraction calculated

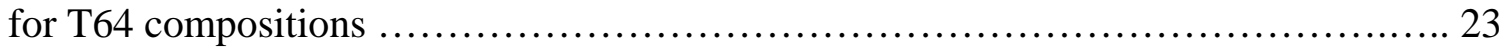

Fig. 4 Values of $1 / \kappa_{1}$ and $1 / \gamma_{1}$ versus alumina volume fraction calculated for AA18 compositions 


\section{Figures -continued}

Fig. 5 Values of $1 / \kappa_{1}$ and $1 / \gamma_{1}$ versus alumina volume fraction calculated for AA5 compositions

Fig. 6 Dependence of $\gamma_{1}$ on alumina reinforcement volume fraction using the values of $B$ determined by the estimation methods discussed and the data fit for compositions using T64 alumina

Fig. 7 Graphical illustrations of the predicted dependence of the effective bulk modulus on reinforcement volume fraction for alumina filled epoxy

Fig. 8 Graphical illustrations of the predicted dependence of the effective shear modulus on reinforcement volume fraction for alumina filled epoxy

Fig. 9 Predicted hydrostatic response for alumina-filled epoxy with an initial alumina volume fraction of $43.3 \%$ 36

Fig. 10 Average normal stresses predicted for large plane-strain compression of an alumina-filled epoxy having an initial alumina volume fraction of $43.4 \%$ 40

Fig. 11 Alumina distention predicted for the large plane-strain compression of an alumina-filled epoxy with an initial alumina volume fraction of $43.4 \%$

Fig. 12 Comparisons of the predicted average normal stress with measurements for large plane-strain compression for several alumina-filled compositions

\section{Tables}

Table 1 Alumina-filled epoxy compositions 17

Table 2 Mass fractions of alumina in compositions examined 20

Table 3 Epoxy and alumina properties 21

Table 4 Strain concentration coefficients for T64 compositions 21

Table 5 Strain concentration coefficients for AA18 compositions 24

Table 6 Strain concentration coefficients for AA5 compositions 25

Table 7 Strain concentration parameters for alumina-filled epoxy compositions 26 


\section{Introduction}

The composition of particle reinforced composite materials can be controlled to provide materials with specific functional attributes. For example, suspending alumina particles in an epoxy matrix produces composite materials exhibiting an increased elastic stiffness relative to the matrix material. A fundamental understanding of the effects of composition and microstructure on the elastic properties of these engineered materials is required for a quantitative analysis of their use in applications. Plate impact experiments ${ }^{1,2}$ have been used to examine the effect of composition, specified as the relative amount and type of alumina particles suspended in the epoxy, on the stress generated during shock-wave compression. It is of interest to be able to predict the stress produced in a specific composition for a given state of strain. For example, the ability to predict the stress intensity of a steady shock-wave as a function of composition would be of great utility in engineering applications involving impact. Currently, there are only two response models ${ }^{1,3}$ that use the basic properties of alumina and epoxy to predict the mechanical response of alumina-filled epoxy, and both models have only been characterized for specific compositions. The purpose of this report is to describe some progress in developing mechanical response models for alumina-filled epoxy that can be used across a range of compositions.

The effect of composition on the mechanical response of alumina-filled epoxy is examined for two situations: the response of the material to small deformations where a linear elastic response is expected and the response of the material to large hydrostatic and plane-strain compressions where nonlinear material response occurs. Measured response data ${ }^{1,2,4,5}$ for compositions containing up to $48 \%$ by volume alumina will be used to develop and test the response models. An outline of this study is described next.

The effect of composition and alumina particle type on the linear elastic response is considered in Section 2. An elastic response model which gives the average stresses and strains in the composite in terms of the volume concentration of the particles and the average stresses and strains in the matrix and particle materials is developed. This model applies to composite materials consisting of perfectly bonded and uniformly distributed particles in a solid isotropic elastic matrix, and uses the elastic properties of the particles and matrix plus three additional parameters, which reflect effects of composition, to characterize a material. Eleven specific compositions formed from three different types of alumina particle are characterized in Section 
3. It is shown that the volume fraction of alumina in the composition has the most significant effect on the elastic response. The effect of particle morphology and size on the elastic response is found to be small for the three particle types examined. Additionally, for a fixed particle type, it is shown that the linear elastic response model for alumina-filled epoxy depends on the bulk and shear moduli of the alumina and epoxy, the alumina volume fraction, and two compositional parameters that are independent of the alumina volume fraction.

The elastic response of alumina-filled epoxy at large compression is nonlinear. ${ }^{1,2,4} \mathrm{~A}$ simple model for response due to the large compression is developed in Section 4. In particular, a nonlinear representation for the epoxy response is introduced and the volume fraction of the alumina in the composition is allowed to increase with compression. The structure of the linear elastic response model is used to define a structural stress for the composition. Motivated by the observation that the stiffness of the alumina is much greater than that of the epoxy, an equilibrium relation between the volume fraction of alumina and invariants of the structural stress is postulated by extending some theoretical work on distended solids. The two parameters needed to calculate the structural stress are determined by matching the bulk and shear modulus of the linear elastic and large compression theories in the limit of small compression. It is found that while the response model for large compression depends explicitly on the alumina volume fraction the other parameters characterizing the model are independent of the alumina volume fraction giving a model that can be applied to a range of compositions. The large compression mechanical response model is applied to hydrostatic and plane-strain compression. Calculated equilibrium Hugoniot states for steady plane-strain shock-waves predicted for a number of compositions formed using a fixed particle type at various initial volume fractions are seen to match measurements remarkably well.

A short summary of the results of this study is given in Section 5 . 


\section{A Model for the Elastic Response for a Discretely Reinforced Composite}

An elastic response model for discretely reinforced solids, developed by Hill, ${ }^{6}$ is used in this study. The model is described below and developed in the context of a uniform isotropic distribution of elastic reinforcing particles suspended in a uniform elastic matrix, and is believed to be representative of the composite materials investigated in this report. Since the original description ${ }^{6}$ was set forth in a general context, the model and details needed for the present study are reviewed below.

\section{A. Model Development}

The model expresses the average macroscopic elastic response of a discretely reinforced composite material in terms of the volume fraction of the reinforcement particles and the average elastic response of the reinforcement and matrix materials. Here it is assumed that the reinforcement and matrix materials can be regarded as isotropic elastic materials and that the reinforcement consists of isolated particles distributed uniformly throughout the matrix material.

The concept of a representative volume of material is fundamental to defining the macroscopic fields and properties appropriate to the composite. ${ }^{6}$ A representative volume contains enough of the matrix and reinforcement to be structurally typical of the composite. When uniform surface tractions and displacements are applied to the surface of the representative volume it is expected that meaningful values for the apparent elastic properties of the composite, reflecting the averages of stresses and strains throughout the volume, can be determined independent of the values of the applied traction and displacement. Consequently, even though the local values of stress and strain may fluctuate rapidly within constituent materials of the composite, well defined average values of stress and strain exist for the composite and contributions from surface irregularities are negligible.

The matrix and reinforcement materials are assumed to be isotropic linear elastic solids with the bulk modulus $K$ and shear modulus $G$ taken as the fundamental pair of elastic constants characterizing the material. The elastic constants for the matrix material will be designated using the subscript ' 1 ' and those for the reinforcement material using the subscript ' 2 '. Elastic constants without a subscript will refer to the macroscopic averages for the composite. Cartesian

tensor notation is used with the subscripted quantities $\sigma_{i j}$ and $\varepsilon_{i j}$ used to represent the components of the stress and strain tensors, respectively, in the rectangular coordinate system. 
The stress and strain of the matrix material will be designated using the superscript '(1)' and for the reinforcement material by the superscript '(2)'.

The average value of a quantity describing the response of the composite material is defined as the integral of that quantity over a region corresponding to a representative volume of the composite divided by the volume of that region. The tilde symbol over a quantity is used to indicate that quantity is an average over the representative volume. It is clear that the average values of the stress and strain in a representative volume are

$$
\tilde{\sigma}_{i j}=c_{1} \tilde{\sigma}_{i j}^{(1)}+c_{2} \tilde{\sigma}_{i j}^{(2)} \text { and } \quad \tilde{\varepsilon}_{i j}=c_{1} \tilde{\varepsilon}_{i j}^{(1)}+c_{2} \tilde{\varepsilon}_{i j}^{(2)}
$$

where $c_{1}$ is the fraction of the representative volume containing the matrix material, $c_{2}$ is the fraction of the representative volume containing the reinforcement material. It is also assumed that $c_{1}$ and $c_{2}$ can be regarded as fixed for small elastic deformations.

The assumption of perfect bonding between the reinforcement particles and matrix implies the sum of volume fractions of matrix and particles equals unity. Consequently, by setting $c_{2}=\varphi$ it follows that

$$
c_{1}=1-\varphi,
$$

allowing (2.1) to be written

$$
\tilde{\sigma}_{i j}=\tilde{\sigma}_{i j}^{(1)}+\varphi\left[\tilde{\sigma}_{i j}^{(2)}-\tilde{\sigma}_{i j}^{(1)}\right] \text { and } \quad \tilde{\varepsilon}_{i j}=\tilde{\varepsilon}_{i j}^{(1)}+\varphi\left[\tilde{\varepsilon}_{i j}^{(2)}-\tilde{\varepsilon}_{i j}^{(1)}\right] .
$$

The relations between stress and strain in the matrix and reinforcement materials are

$$
\sigma_{i j}^{(1)}=\left(K_{1}-\frac{2}{3} G_{1}\right) \varepsilon_{k k}^{(1)} \delta_{i j}+2 G_{1} \varepsilon_{i j}^{(1)} \quad \text { and } \quad \sigma_{i j}^{(2)}=\left(K_{2}-\frac{2}{3} G_{2}\right) \varepsilon_{k k}^{(2)} \delta_{i j}+2 G_{2} \varepsilon_{i j}^{(2)} .
$$

Because the distribution of reinforcement particles in the representative volume of the composite is uniform and isotropic, the stress-strain relations given by (2.4) also hold for the averages of the stress and strain in each component of the composite giving

$$
\tilde{\sigma}_{i j}^{(1)}=\left(K_{1}-\frac{2}{3} G_{1}\right) \tilde{\varepsilon}_{k k}^{(1)} \delta_{i j}+2 G_{1} \tilde{\varepsilon}_{i j}^{(1)} \quad \text { and } \quad \tilde{\sigma}_{i j}^{(2)}=\left(K_{2}-\frac{2}{3} G_{2}\right) \tilde{\varepsilon}_{k k}^{(2)} \delta_{i j}+2 G_{2} \tilde{\varepsilon}_{i j}^{(2)} .
$$

Equations (2.3) and (2.5) provide relations between average field quantities in the composite and each of its component materials. However, additional information relating the average stress and strain describing the composite to corresponding quantities of the components is required 
before the elastic response of the composite can be specified in terms of the reinforcement volume fraction and elastic properties of the matrix and reinforcement materials. Suppose that in equilibrium the average strains in the matrix and particles are uniquely related to the average strain for the representative volume according to the transformations

$$
\tilde{\varepsilon}_{i j}^{(1)}=A_{i j k l}^{(1)} \tilde{\varepsilon}_{k l} \quad \text { and } \quad \tilde{\varepsilon}_{i j}^{(2)}=A_{i j k l}^{(2)} \tilde{\varepsilon}_{k l} .
$$

The tensors $A_{i j k l}^{(1)}$ and $A_{i j k l}^{(2)}$ reflect characteristics of the composite and will also depend on the elastic properties of the component materials, volume concentration of the reinforcement phase, and microstructural arrangement of reinforcement particles in the matrix. Substituting (2.6) into the second of (2.3) provides a specific constraint between the components of $A_{i j k l}^{(1)}$ and $A_{i j k l}^{(2)}$ through the relation

$$
(1-\varphi) A_{i j k l}^{(1)}+\varphi A_{i j k l}^{(2)}=\delta_{i k} \delta_{j l} .
$$

The symbol $\delta_{i j}$ is known as the Kronecker delta and has the properties that it vanishes whenever $i \neq j$ and equals unity otherwise. Requiring that the average stresses and strains of the composite remain isotropic requires that $A_{i j k l}^{(1)}$ and $A_{i j k l}^{(2)}$ be fourth-order isotropic tensors. Since $\tilde{\varepsilon}_{i j}=\tilde{\varepsilon}_{j i}$, the simplest representations ${ }^{7}$ for $A_{i j k l}^{(1)}$ and $A_{i j k l}^{(2)}$ allowed that satisfy the isotropy requirement are

$$
A_{i j k l}^{(1)}=\left(\kappa_{1}-\frac{2}{3} \gamma_{1}\right) \delta_{i j} \delta_{k l}+2 \gamma_{1} \delta_{i k} \delta_{j l} \quad \text { and } \quad A_{i j k l}^{(2)}=\left(\kappa_{2}-\frac{2}{3} \gamma_{2}\right) \delta_{i j} \delta_{k l}+2 \gamma_{2} \delta_{i k} \delta_{j l} \text {. }
$$

The parameters $\kappa_{1}, \kappa_{2}, \gamma_{1}$, and $\gamma_{2}$ are called strain concentration coefficients and reflect effects on the composite response due to differences in elastic properties of the constituent materials, volume concentration of the reinforcement, and microstructural arrangement of the reinforcement particles in the matrix. Substituting (2.8) into (2.6) gives

$$
\tilde{\varepsilon}_{i j}^{(1)}=\left(\kappa_{1}-\frac{2}{3} \gamma_{1}\right) \tilde{\varepsilon}_{k k} \delta_{i j}+2 \gamma_{1} \tilde{\varepsilon}_{i j} \quad \text { and } \quad \tilde{\varepsilon}_{i j}^{(2)}=\left(\kappa_{2}-\frac{2}{3} \gamma_{2}\right) \tilde{\varepsilon}_{k k} \delta_{i j}+2 \gamma_{2} \tilde{\varepsilon}_{i j} \cdot
$$

The constraint of (2.7) yields

$$
(1-\varphi) \kappa_{1}+\varphi \kappa_{2}=\frac{1}{3} \text { and }(1-\varphi) \gamma_{1}+\varphi \gamma_{2}=\frac{1}{2}
$$


Equations (2.10) show that only one of the parameters sets $\left(\kappa_{1}, \kappa_{2}\right)$ and $\left(\gamma_{1}, \gamma_{2}\right)$ is independent. On selecting the parameter pair $\left(\kappa_{1}, \gamma_{1}\right)$ as independent, the other pair $\left(\kappa_{2}, \gamma_{2}\right)$ is given by

$$
\kappa_{2}=\kappa_{1}+\left(1-3 \kappa_{1}\right) / 3 \varphi \text { and } \quad \gamma_{2}=\gamma_{1}+\left(1-2 \gamma_{1}\right) / 2 \varphi
$$

It is useful to note the limiting behavior of the strain concentration factors as the volume fraction of the reinforcement vanishes and the composite becomes entirely composed of the matrix material. The average strain in the matrix and composite material must then be equal since there is no reinforcement. Consequently, (2.10) gives $3 \kappa_{1} \rightarrow 1$ and $2 \gamma_{1} \rightarrow 1$ as $\varphi \rightarrow 0$. At the other limit where $\varphi \rightarrow 1$, the average strain in the reinforcement phase and composite must be identical since the matrix material is absent. Consequently, (2.10) implies that $3 \kappa_{2} \rightarrow 1$ and $2 \gamma_{2} \rightarrow 1$ as $\varphi \rightarrow 1$.

Equations (2.3), (2.5), (2.9), and (2.11) provide an equilibrium model relating the average stress to the average strain in a discretely reinforced composite material.

\section{B. Parameter Characterization}

In order to use the model described above, the volume fraction, elastic properties of the particle and matrix materials, and two strain concentration coefficients need to be known. It is supposed that the reinforcement and matrix materials are well characterized. Consequently, the densities and elastic properties of the particle and matrix materials are known.

Let $\rho_{1}$ and $\rho_{2}$ denote the densities of the matrix and reinforcement materials, respectively. If it is assumed that there is no significant residual stress in the composite, the average densities $\tilde{\rho}_{1}$ and $\tilde{\rho}_{2}$ of the matrix and reinforcement materials, respectively, can be taken as the actual densities of the corresponding constituent materials used to form the composite. The volume fraction of the particles can be determined from measurement of the average density, $\tilde{\rho}$, of the composite material through the relation

$$
\varphi=\left(\tilde{\rho}-\tilde{\rho}_{1}\right) /\left(\tilde{\rho}_{2}-\tilde{\rho}_{1}\right)
$$

Specification of the two strain concentration coefficients, $\kappa_{1}$ and $\gamma_{1}$, then completes the parameter characterization for the composite material. As mentioned above, these two 
coefficients are expected to reflect the effects on the composite response due to differences in elastic properties of the component materials, volume concentration of the reinforcement, and microstructural arrangement of the reinforcement particles in the matrix. These two coefficients may be determined from the effective elastic properties of the composite. It is possible that composite materials comprised of isotropic elastic constituents may not necessarily be also isotropic. For example, a composite material might be constructed with a regular cubic array of reinforcement particles so that the macroscopic response of a representative volume would be expected to have the symmetry of a cubic crystal. However, it is assumed here that the matrix contains a random uniform isotropic distribution of reinforcement particles so that the expected response of a representative volume of the composite is also isotropic. The relation between the apparent stress and strain of a representative volume will then be

$$
\tilde{\sigma}_{i j}=\left(K-\frac{2}{3} G\right) \tilde{\varepsilon}_{k k} \delta_{i j}+2 G \tilde{\varepsilon}_{i j} .
$$

Expressions for the effective elastic properties of the composite may be obtained by substituting (2.5), with (2.9), into the first of (2.1) yielding

$$
\tilde{\sigma}_{i j}=\left\{3\left(c_{1} \kappa_{1} K_{1}+c_{2} \kappa_{2} K_{2}\right)-\frac{4}{3}\left(c_{1} \gamma_{1} G_{1}+c_{2} \gamma_{2} G_{2}\right)\right\} \tilde{\varepsilon}_{k k} \delta_{i j}+4\left(c_{1} \gamma_{1} G_{1}+c_{2} \gamma_{2} G_{2}\right) \tilde{\varepsilon}_{i j} .
$$

Comparison of (2.13) and (2.14) yields the following correspondence between the effective elastic properties of the composite and the parameters appearing in the elastic response model

$$
K=3\left(c_{1} \kappa_{1} K_{1}+c_{2} \kappa_{2} K_{2}\right), \quad G=2\left(c_{1} \gamma_{1} G_{1}+c_{2} \gamma_{2} G_{2}\right) \text {. }
$$

Using the constraints of (2.2) and (2.10) in (2.15) gives

$$
K=K_{2}+3(1-\varphi) \kappa_{1}\left(K_{1}-K_{2}\right) \quad \text { and } \quad G=G_{2}+2(1-\varphi) \gamma_{1}\left(G_{1}-G_{2}\right) .
$$

The two independent strain concentration coefficients needed to complete characterization of the response model can therefore be written

$$
\kappa_{1}=\frac{1}{3(1-\varphi)} \frac{\left(K-K_{2}\right)}{\left(K_{1}-K_{2}\right)} \text { and } \gamma_{1}=\frac{1}{2(1-\varphi)} \frac{\left(G-G_{2}\right)}{\left(G_{1}-G_{2}\right)}
$$

and determined from measured values for the elastic moduli of a particular composite. 


\section{Strain Concentration Coefficient Estimates}

The elastic response model for composites in the form (2.13) is widely used since it usually convenient to measure the effective elastic properties used in (2.13) directly. However, many situations arise in which it is desired to fabricate a composite material with specific effective elastic properties and a reasonably accurate methodology for estimating the effective elastic properties is then desired. Most practical methods for providing these estimates for isotropic

elastic materials are based on using (2.16) with various approximations for $\kappa_{1}$ and $\gamma_{1} .8$ Several of the common approximations for the strain concentration factors that find use in providing estimates for the effective elastic properties of isotropic elastic composites will now be described.

One common method often used to predict the effective elastic properties of a composite is called the rule of mixtures. The method follows from the assumption that the average strains in the reinforcement and matrix materials are the same as the average macroscopic strain applied to the composite. When the average strains are identical, $\tilde{\varepsilon}_{i j}^{(1)}=\tilde{\varepsilon}_{i j}^{(2)}=\tilde{\varepsilon}_{i j}$, (2.9) is satisfied for all volume fractions of reinforcement material by

$$
\kappa_{1}^{\varepsilon}=\frac{1}{3} \text { and } \quad \gamma_{1}^{\varepsilon}=\frac{1}{2} \text {. }
$$

The superscript ' $\varepsilon$ ' indicates that the values of $\kappa_{1}$ and $\gamma_{1}$ in (2.18) result from the assumption of identical average strains. It is clear that this assumption also implies $\kappa_{2}^{\varepsilon}=1 / 3$ and $\gamma_{2}^{\varepsilon}=1 / 2$. Using (2.18) in (2.16) yields the rule of mixtures for predicting the effective elastic properties of the composite

$$
K^{\varepsilon}=(1-\varphi) K_{1}+\varphi K_{2} \quad \text { and } \quad G^{\varepsilon}=(1-\varphi) G_{1}+\varphi G_{2} .
$$

An alternate method that is often used to predict the effective elastic properties of a composite is called the inverse rule of mixtures and follows from the assumption that the average stresses in the reinforcement and matrix materials are the same as the average macroscopic stress applied to the composite. Assuming that $\tilde{\sigma}_{i j}^{(1)}=\tilde{\sigma}_{i j}^{(2)}=\tilde{\sigma}_{i j}$, (2.5) and (2.9) can be combined to show, for all possible values of $\varphi$, that $\kappa_{1} K_{1}=\kappa_{2} K_{2}$ and $\gamma_{1} G_{1}=\gamma_{2} G_{2}$. Using (2.11) with this result gives

$$
\kappa_{1}^{\sigma}=\frac{1}{3\left[(1-\varphi)+\varphi\left(K_{1} / K_{2}\right)\right]} \quad \text { and } \quad \gamma_{1}^{\sigma}=\frac{1}{2\left[(1-\varphi)+\varphi\left(G_{1} / G_{2}\right)\right]} \text {. }
$$


The superscript ' $\sigma$ ' indicates that the values of $\kappa_{1}$ and $\gamma_{1}$ in (2.20) result from the assumption of identical average stresses. Substituting (2.20) into (2.16) yields the inverse rule of mixtures for predicting the effective elastic properties of composites

$$
\frac{1}{K^{\sigma}}=\frac{(1-\varphi)}{K_{1}}+\frac{\varphi}{K_{2}} \text { and } \quad \frac{1}{G^{\sigma}}=\frac{(1-\varphi)}{G_{1}}+\frac{\varphi}{G_{2}} .
$$

A useful approximation for the effective moduli of composites containing a dilute suspension of particles can be obtained as follows. If the volume fraction of the particles is small, then a linear transformation of the form (2.6) provides an approximate relation between the average strain field characterizing the configuration of a particles and the average strain field of the matrix phase according to

$$
\tilde{\varepsilon}_{i j}^{(2)}=\frac{1}{3}(a-b) \tilde{\varepsilon}_{k k}^{(1)} \delta_{i j}+b \tilde{\varepsilon}_{i j}^{(1)} .
$$

In the special case of a spherical isotropic elastic particle embedded in another isotropic elastic material with an applied uniform strain far from the particle of $\tilde{e}_{i j}^{(1)}$, it has been shown that the strain in the particle is uniform and that ${ }^{9}$

$$
a=\frac{3 K_{1}+4 G_{1}}{3 K_{2}+4 G_{1}} \quad \text { and } \quad b=\frac{5 G_{1}\left(3 K_{1}+4 G_{1}\right)}{G_{1}\left(9 K_{1}+8 G_{1}\right)+6 G_{2}\left(K_{1}+2 G_{1}\right)} .
$$

Using (2.7) and the second of (2.3) gives

$$
\tilde{\varepsilon}_{i j}=\left\{\frac{1}{3}(a-b) \varphi\right\} \tilde{\varepsilon}_{k k}^{(1)} \delta_{i j}+\{1+(b-1) \varphi\} \tilde{\varepsilon}_{i j}^{(1)} .
$$

The first of (2.9) and (2.24) are compatible provided that

$$
3 \kappa_{1}=\frac{1}{[1+(a-1) \varphi]} \text { and } 2 \gamma_{1}=\frac{1}{[1+(b-1) \varphi]} \text {. }
$$

Substituting (2.25) into (2.16) gives the following estimates for the effective moduli

$$
K^{D}=\frac{(1-\varphi) K_{1}+a \varphi K_{2}}{[1+(a-1) \varphi]} \text { and } G^{D}=\frac{(1-\varphi) G_{1}+b \varphi G_{2}}{[1+(b-1) \varphi]} .
$$

The superscript " $D$ " indicates these estimates were obtained by assuming a dilute suspension of particles in the matrix. In the case of spherical particles, (2.23) and (2.26) provide the following estimates, accurate to first order in the volume fraction of the particles, for the effective bulk 
modulus, compressibility $\left(1 / K^{D}\right)$, and shear modulus for the composite material containing a diffuse distribution of particles:

$$
\begin{aligned}
& K^{D}=K_{1}+a\left(K_{2}-K_{1}\right) \varphi=K_{1}+\frac{\left(3 K_{1}+4 G_{1}\right)}{\left(3 K_{2}+4 G_{1}\right)}\left(K_{2}-K_{1}\right) \varphi \\
& \frac{1}{K^{D}}=\frac{1}{K_{1}}+\frac{\left(3+4 G_{1} / K_{1}\right)}{\left(3+4 G_{1} / K_{2}\right)}\left(\frac{1}{K_{2}}-\frac{1}{K_{1}}\right) \varphi \\
& G^{D}=G_{1}+b\left(G_{2}-G_{1}\right) \varphi=G_{1}\left\{1+\frac{5\left(3 K_{1}+4 G_{1}\right)\left(G_{2}-G_{1}\right)}{\left[6 G_{2}\left(K_{1}+2 G_{1}\right)+G_{1}\left(9 K_{1}+8 G_{1}\right)\right]} \varphi\right\} .
\end{aligned}
$$

The expressions for the effective compressibility and shear modulus given in (2.27) match the results for a dilute suspension of spherical particles obtained earlier using a different derivation. ${ }^{10}$

The rule of mixtures and inverse rule of mixtures find application in special situations such as layered composite structures. However, as pointed out by Hill, ${ }^{6}$ neither assumption is valid for the types of composites considered here. The predicted values for the elastic properties using the rule of mixtures will provide an upper bound on the actual values while values predicted using the inverse rule of mixtures will provide a lower bound. These bounds on the elastic properties of the composite, and corresponding values for $\kappa_{1}$ and $\gamma_{1}$, might be of some use when the elastic properties of the particle and matrix materials are similar. But, when the elastic moduli of the particle and matrix materials differ greatly, as is the case for the materials investigated here, the upper and lower bounds on the elastic moduli are significantly different.

The elastic properties for a number of composite compositions containing one of three types of alumina powder reinforcement suspended in a Z-hardened Epon 828 matrix material have been measured. ${ }^{2}$ The model parameters described above will be determined for each of these compositions in the next section. It is seen that the model formulation above proves useful in identifying systematic dependencies of the strain concentration coefficients on alumina volume concentration and particle type for this family of composite materials. 


\section{Characterization of Several Alumina-Filled Epoxy Compositions}

Table 1 lists the alumina-filled epoxy compositions examined in the present study. These materials were fabricated by suspending various amounts of one of three types of alumina particle in a common epoxy formulation consisting of Epon 828 resin $^{11}$ and Epi-Cure $\mathrm{Z}$ curing agent ${ }^{12}$ mixed 5:1 by weight. The first column identifies the type of alumina particle used in the composition. Five compositions use T64 alumina ${ }^{13}$ which is made from irregular shaped particles ranging in size from approximately 2 to $30 \mu \mathrm{m}$. There are four compositions using AA18 alumina ${ }^{14}$ which consists of faceted but roughly spherical particles that have a nominal diameter distribution of $18 \pm 5 \mu \mathrm{m}$. The final two compositions listed use AA5 alumina ${ }^{14}$ which is similar to AA18 in shape, but has a nominal diameter distribution of $5 \pm 2 \mu \mathrm{m}$.

Table 1. Alumina-filled epoxy compositions.

\begin{tabular}{|c|c|c|c|c|c|}
\hline $\begin{array}{c}\text { Alumina } \\
\text { Type }\end{array}$ & $\begin{array}{c}\tilde{\rho} \\
\left(\mathrm{kg} / \mathrm{m}^{3}\right)\end{array}$ & $\begin{array}{c}C_{L} \\
(\mathrm{~m} / \mathrm{s})\end{array}$ & $\begin{array}{c}C_{S} \\
(\mathrm{~m} / \mathrm{s})\end{array}$ & $\begin{array}{c}K \\
(\mathrm{GPa})\end{array}$ & $\begin{array}{c}G \\
(\mathrm{GPa})\end{array}$ \\
\hline $\mathrm{T} 64^{\mathrm{a}}$ & 1750 & 2738 & 1336 & 8.95 & 3.12 \\
\hline T64 & 2121 & 2987 & 1558 & 12.1 & 5.15 \\
\hline T64 & 2233 & 3033 & 1592 & 13.0 & 5.66 \\
\hline T64 & 2377 & 3197 & 1720 & 14.9 & 7.03 \\
\hline T64 & 2496 & 3314 & 1823 & 16.4 & 8.30 \\
\hline AA18 ${ }^{b}$ & 1760 & 2677 & 1308 & 8.60 & 3.01 \\
\hline AA18 & 2242 & 3009 & 1593 & 12.7 & 5.69 \\
\hline AA18 & 2389 & 3132 & 1701 & 14.2 & 6.91 \\
\hline AA18 & 2525 & 3295 & 1825 & 16.2 & 8.41 \\
\hline $\mathrm{AA} 5^{\mathrm{c}}$ & 1765 & 2635 & 1276 & 8.42 & 2.87 \\
\hline AA5 & 2391 & 3034 & 1585 & 14.0 & 6.01 \\
\hline
\end{tabular}

${ }^{\mathrm{a}}$ T64 tabular alumina has irregularly shaped particles with dimensions ranging from approximately 2 to $30 \mu \mathrm{m}$.

${ }^{\mathrm{b}}$ AA18 alumina has faceted roughly spherically shaped particles $18 \pm 5 \mu \mathrm{m}$ in diameter.

${ }^{\mathrm{c}}$ AA5 alumina has faceted roughly spherically shaped particles $5 \pm 2 \mu \mathrm{m}$ in diameter. 


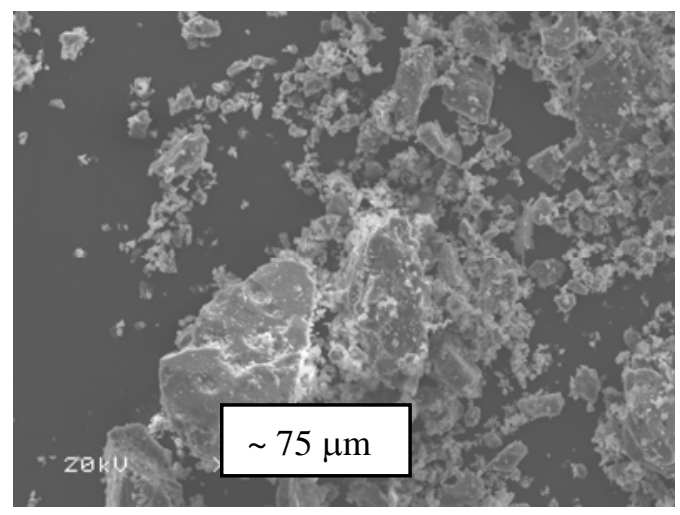

T64 Alumina Particles

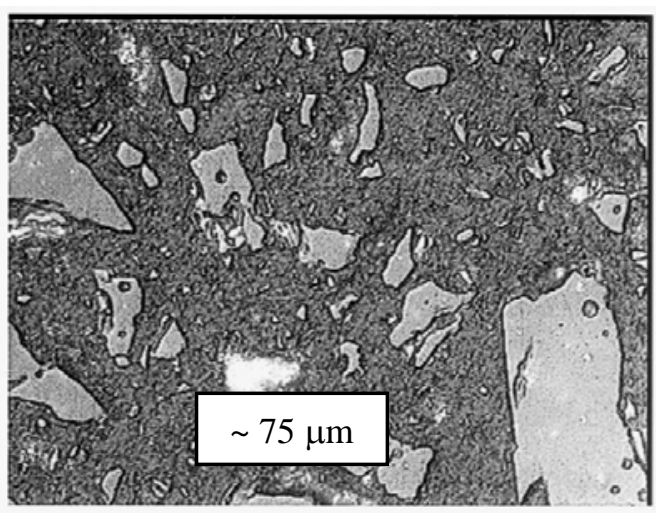

T64 Alumina in Epoxy

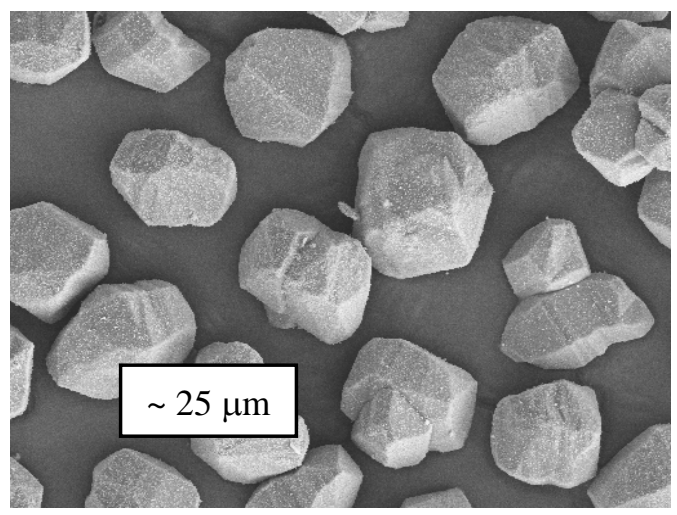

AA18 Alumina Particles

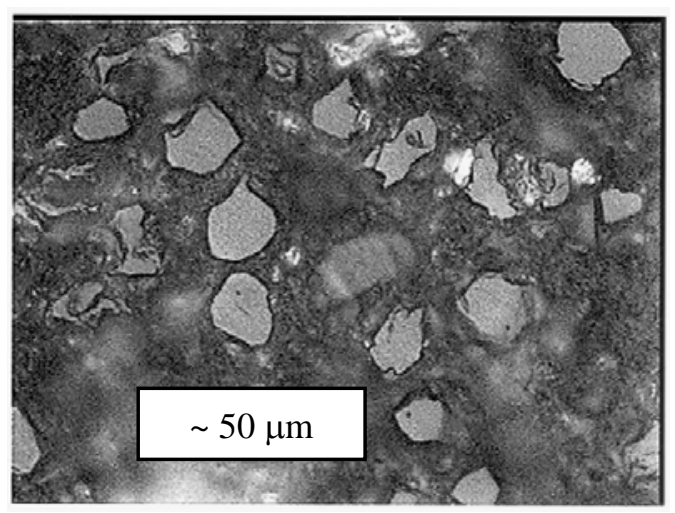

AA18 Alumina in Epoxy

Figure 1. Microscope images of T64 and AA18 alumina particles above the corresponding images of polished surfaces of compositions, containing roughly $43 \%$ alumina by volume, fabricated from these two particle types.

Figure 1 shows microscope images of the T64 and AA18 alumina particles above images of polished surfaces of composites fabricated containing roughly $43 \%$ by volume of the corresponding alumina particle type.

Each composition listed in Table 1 was fabricated using the same mixing and curing steps. Constituents were mixed after being heated to $71^{\circ} \mathrm{C}$, then vacuum out-gassed before and after being poured into heated cylindrical molds. Curing of all compositions used a schedule of $6 \mathrm{~h}$ at $54^{\circ} \mathrm{C}$ followed by $16 \mathrm{~h}$ at $93^{\circ} \mathrm{C}$ achieved using a $6 \mathrm{~h}$ ramp and final cooling over $5 \mathrm{~h}$ to room temperature. Cylindrical billets nominally $64 \mathrm{~mm}$ in diameter by $130 \mathrm{~mm}$ long were fabricated for each composition. A number of sample disks having diameter $50.8 \mathrm{~mm}$ and thickness ranging from 2.0-20.0 mm thick were cut from the billets for characterization. The second column in Table 1 gives the measured density obtained by measuring and weighing at least five samples of each composition. The third and fourth columns list average values for the longitudinal and shear 
wave speeds for each composition. These wave speeds were determined from pulse-echo, timeof-flight measurements using $5 \mathrm{MHz}$ ultrasonic longitudinal and shear transducers coupled to sample surfaces perpendicular to an axis defined by gravity for the cast materials. The final two columns list the effective bulk and shear modulus calculated according to

$$
K=\rho\left[c_{L}^{2}-4 c_{S}^{2} / 3\right] \text { and } G=\rho c_{S}^{2}
$$

for each composition using the values in columns 2, 3, and 4.

\section{A. Average Elastic Properties of the Epoxy Matrix and Alumina Reinforcement}

Sample disks were also cut from billets of epoxy fabricated using the Epon 828 resin and EpiCure Z curing agent mixed 5:1 by weight. These billets of unfilled epoxy were nominally $64 \mathrm{~mm}$ in diameter and approximately $50 \mathrm{~mm}$ in length. The short length of these billets was needed to reduce excess heating of the billet due to the exothermic cure of this epoxy system. An average density of $1,203 \mathrm{~kg} / \mathrm{m}^{3}$ was determined for the unfilled epoxy by calculation using the measured weight and dimensions of at least five samples. Longitudinal and shear wave speeds were measured using the ultrasonic method described above and yielded an average longitudinal wave speed of 2,645 m/s and average shear wave speed of 1,210 m/s based on measurements at five locations on three samples. These measurements result in calculated bulk and shear moduli, using (3.1), of 6.07 GPa and 1.76 GPa, respectively.

The characteristics of the different alumina powder used to fabricate the compositions examined in this study could not be measured directly. The T64 alumina powder is fabricated by crushing and sieving alumina ceramic sintered at high temperature while the AA18 and AA5 powder is formed by high temperature vapor deposition with the nominal average diameter of the particle is controlled by the duration of deposition. Consequently, it was suspected that the average density of the T64 alumina powder might differ from the average densities of the AA18 and AA5 powders. Because the masses of the constituents for each composition were measured, estimates of the density of each alumina type and volume fraction can be established. Let $\tilde{\rho}_{E}$ and $\tilde{\rho}_{A}$ be the average densities of the epoxy matrix and the alumina powder respectively. The mass fractions $m$ of alumina for each composition can be calculated using the measured weights of the constituents used in fabrication. If it is assumed that vacuum out-gassing eliminates all void space, the following relation between densities of the epoxy and alumina holds 


$$
\left[\frac{1}{\tilde{\rho}_{E}}-\frac{1}{\tilde{\rho}}\right]=m\left[\frac{1}{\tilde{\rho}_{E}}-\frac{1}{\tilde{\rho}_{A}}\right],
$$

and can be used to calculate the average density of alumina powder in each composition. Equation (2.12) can then be used to calculate the volume fraction of alumina powder in each composition.

Equation (3.2) was used to provide estimates of the average density for the alumina powders used in this study. The first three columns of Table 2 list the type of alumina powder and the masses of the alumina powder and epoxy (Epon 828 resin plus Z-hardener) recorded during fabrication of the billets for compositions with an alumina volume fraction greater than $30 \%$. The fourth column gives the mass fraction of alumina in the composition having the measured density reported in column five.

Table 2. Mass fractions of alumina in compositions examined.

\begin{tabular}{|c|c|c|c|c|}
\hline \multicolumn{2}{|c|}{ Alumina } & \multirow{2}{*}{$\begin{array}{l}\text { Epoxy } \\
\mathrm{M}_{\mathrm{E}}(\mathrm{g})\end{array}$} & \multirow[t]{2}{*}{$m$} & \multirow{2}{*}{$\begin{array}{c}\tilde{\rho} \\
\left(\mathrm{kg} / \mathrm{m}^{3}\right)\end{array}$} \\
\hline Type & $\mathrm{M}_{\mathrm{A}}(\mathrm{g})$ & & & \\
\hline T64 & 1600.5 & 960.5 & 0.6250 & 2120 \\
\hline $\mathrm{T} 64$ & 1680.5 & 840.2 & 0.6667 & 2233 \\
\hline T64 & 1800.6 & 719.9 & 0.7144 & 2377 \\
\hline T64 & 1800.2 & 600.1 & 0.7500 & 2496 \\
\hline AA18 & 1680.1 & 840.0 & 0.6667 & 2242 \\
\hline AA18 & 1800.1 & 720.3 & 0.7142 & 2389 \\
\hline AA18 & 1800.3 & 600.2 & 0.7500 & 2525 \\
\hline AA5 & 1799.9 & 720.0 & 0.7143 & 2391 \\
\hline
\end{tabular}

Using equation (3.2) to form a least squares fit to the values listed in Table 2 that is constrained to pass through the limiting value of density for the pure epoxy, gives the values 3,899 and $3,960 \mathrm{~kg} / \mathrm{m}^{3}$ for the average densities of the T64 and AA18 alumina powders, respectively. The calculated density of each T64 and AA18 sample varies less than $\pm 1 \%$ from the corresponding average values determined from the least squares fit. An average powder density 
of $3,957 \mathrm{~kg} / \mathrm{m}^{3}$ was found for the two AA5 powder data points and, as expected, is close to the average density found for the AA18 powder.

The elastic properties of the alumina particles are estimated since was impractical to perform measurements due to the small size. The elastic properties for $\alpha$-alumina were taken as representative since it has a density ${ }^{1}$ of $3,969 \mathrm{~kg} / \mathrm{m}^{3}$ that is reasonable close to the values determined here for the three types of alumina used in this study. Table 3 lists the average density and elastic properties used for the matrix and alumina reinforcement materials.

Table 3. Epoxy and alumina properties.

\begin{tabular}{cccccc}
\hline \hline Material & $\tilde{\rho}$ & $c_{L}$ & $c_{S}$ & $K$ & $G$ \\
$\left(\mathrm{~kg} / \mathrm{m}^{3}\right)$ & $(\mathrm{m} / \mathrm{s})$ & $(\mathrm{m} / \mathrm{s})$ & $\begin{array}{c}(\mathrm{GPa}) \\
(\mathrm{GPa})\end{array}$ \\
\hline Epoxy $^{\mathrm{a}}$ & 1203 & 2645 & 1210 & 6.07 & 1.76 \\
Alumina $^{\mathrm{b}}$ & 3969 & - & - & 263 & 160 \\
\hline \hline
\end{tabular}

${ }^{a}$ Average density and sound speeds measured on samples prepared for this study.

${ }^{\mathrm{b}}$ Density and elastic moduli from Munson, Boade, and Schuler. ${ }^{1}$

\section{B. Strain Concentration Coefficients for T64 Compositions}

Table 4 lists density, elastic moduli, reinforcement volume fraction, and strain concentration coefficients for the T64 compositions. The reinforcement volume fraction was calculated using

Table 4. Strain concentration coefficients for T64 compositions.

\begin{tabular}{cccccc}
\hline \hline $\begin{array}{c}\rho \\
\left(\mathrm{kg} / \mathrm{m}^{3}\right)\end{array}$ & $\begin{array}{c}\boldsymbol{K} \\
(\%)\end{array}$ & $\begin{array}{c}\text { (GPa) } \\
(\mathrm{GPa})\end{array}$ & $\kappa_{1}$ & $\gamma_{1}$ \\
\hline 1750 & 20.3 & 8.95 & 3.12 & 0.414 & 0.622 \\
2121 & 33.9 & 12.1 & 5.15 & 0.494 & 0.742 \\
2233 & 38.1 & 13.0 & 5.66 & 0.525 & 0.789 \\
2377 & 43.4 & 14.9 & 7.03 & 0.570 & 0.856 \\
2496 & 47.8 & 16.4 & 8.30 & 0.615 & 0.921 \\
\hline \hline
\end{tabular}


(2.12) with the values of average density for the epoxy and alumina from Table 3 and where (2.17) was used to calculate the strain concentration coefficients.

Figure 2 shows the effect of alumina volume fraction on the strain concentration coefficients for the T64 compositions. Quadratic fits to the values of the strain concentration coefficients, constrained to pass through the appropriate limiting values at $\varphi=0$, giving

$$
\begin{aligned}
& \kappa_{1}^{T 64}=\frac{1}{3}\left[1+0.674 \varphi+2.223 \varphi^{2}\right] \\
& \gamma_{1}^{T 64}=\frac{1}{2}\left[1+0.712 \varphi+2.124 \varphi^{2}\right]
\end{aligned}
$$

are shown in Figure 2, and provide reasonable approximations to the coefficients for the T64 composition over the range of reinforcement volume fraction examined.

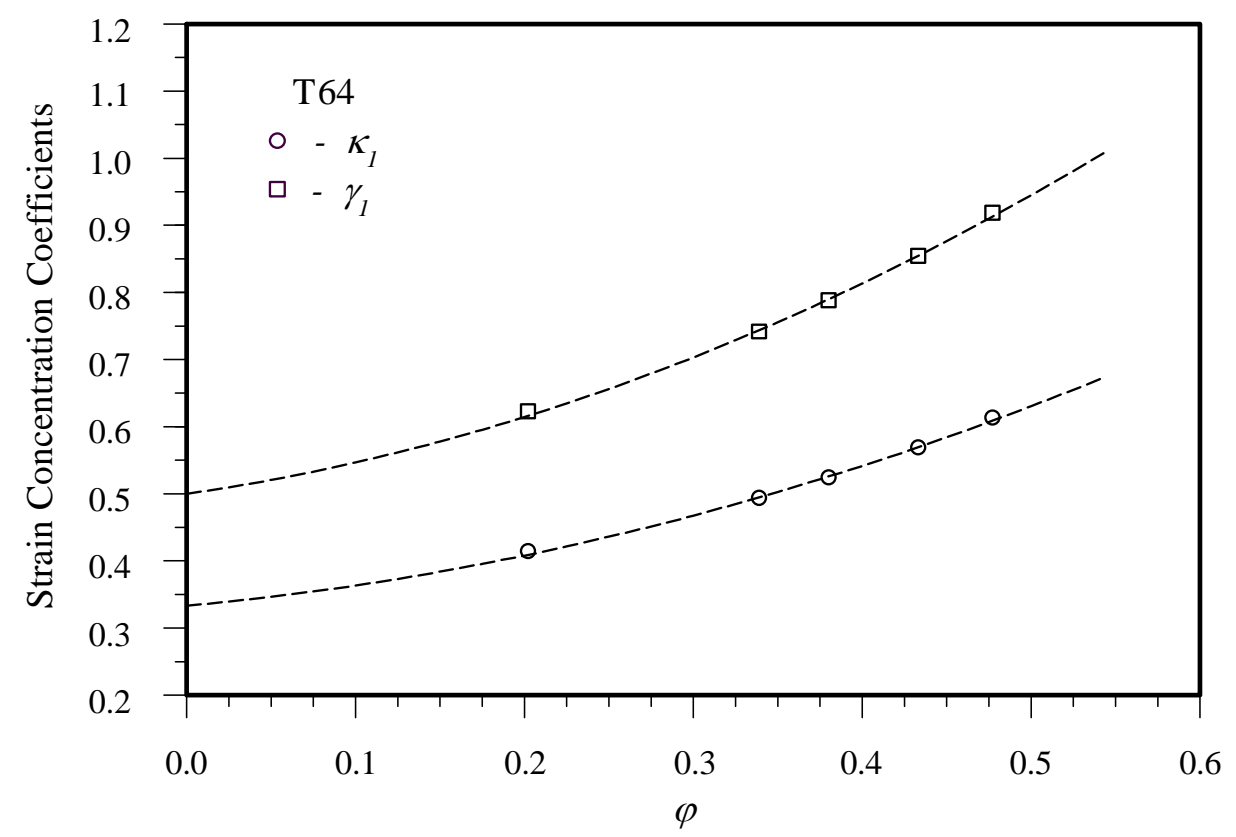

Figure 2. Values of $\kappa_{1}$ and $\gamma_{1}$ versus alumina volume fraction calculated from measured acoustic speeds and density for T64 compositions. The curves shown are second order polynomial fits constrained to pass through appropriate values for each coefficient at $\varphi=0$. 
An approximation to the two strain concentration coefficients for the T64 compositions involving a single parameter can be obtained by fitting the inverse of the strain concentration coefficients to the functional form

$$
\frac{1}{\kappa_{1}}=3[1+A \varphi] \text { and } \frac{1}{\gamma_{1}}=2[1+B \varphi]
$$

suggested by (2.25), where the terms $(a-1)$ and $(b-1)$ in (2.25) are replaced with $A$ and $B$, respectively.

Figure 3 is a plot showing the inverses of the strain concentration coefficients determined for the T64 compositions examined. A linear least squares fit, constrained to pass through the appropriate limiting values at $\varphi=0$, to the inverse values of the strain concentration coefficients as a function of the reinforcement volume fraction is also shown in Figure 3, and is seen to provide a good fit to the data with $A_{T 64}=-0.9545$ and $B_{T 64}=-0.9564$.

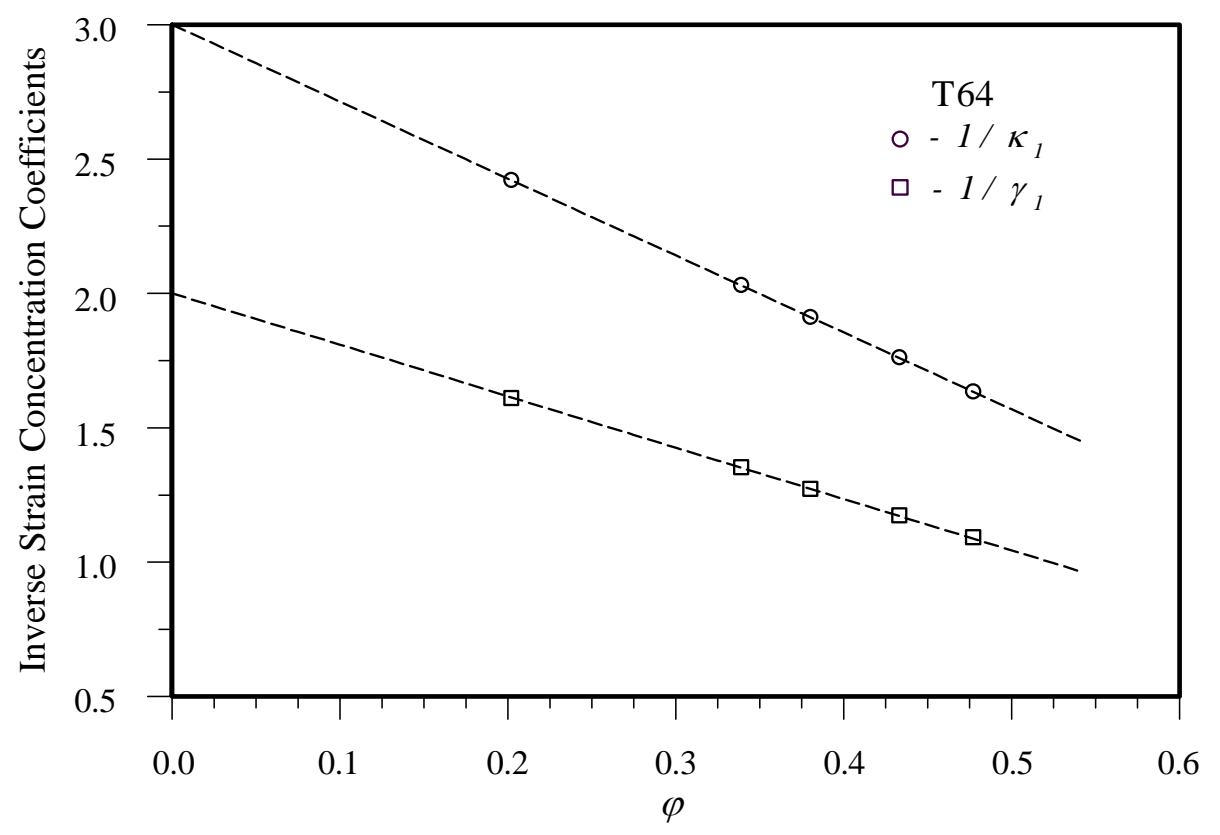

Figure 3. Values of $1 / \kappa_{1}$ and $1 / \gamma_{1}$ versus alumina volume fraction calculated for T64 compositions. The curves shown are linear fits constrained to pass through appropriate values for each coefficient at $\varphi=0$. 


\section{Strain Concentration Coefficients for AA18 Compositions}

Table 5 provides a listing of the reinforcement volume fraction and strain concentration coefficients determined for the AA18 compositions examined. A plot of the inverses of these strain concentration coefficients as a function of alumina volume fraction is shown in Figure 4. A linear least squares fit, constrained to pass through the appropriate limiting values at $\varphi=0$, as a function of the reinforcement volume fraction was again performed and is shown in Figure 4. The least square fits to these points gives $A_{A A 18}=-0.9579$ and $B_{A A 18}=-0.9574$.

Table 5. Strain concentration coefficients for AA18 compositions.

\begin{tabular}{cccccc}
\hline \hline $\begin{array}{c}\rho \\
\left(\mathrm{kg} / \mathrm{m}^{3}\right)\end{array}$ & $\begin{array}{c}\varphi \\
(\%)\end{array}$ & $\begin{array}{c}\text { (GPa) } \\
1760\end{array}$ & $\begin{array}{c}G \\
(\mathrm{GPa})\end{array}$ & $\kappa_{1}$ & $\gamma_{1}$ \\
2242 & 20.2 & 8.60 & 3.01 & 0.413 & 0.620 \\
2389 & 37.6 & 12.7 & 5.69 & 0.524 & 0.786 \\
2525 & 42.9 & 14.2 & 5.91 & 0.566 & 0.849 \\
\hline \hline
\end{tabular}

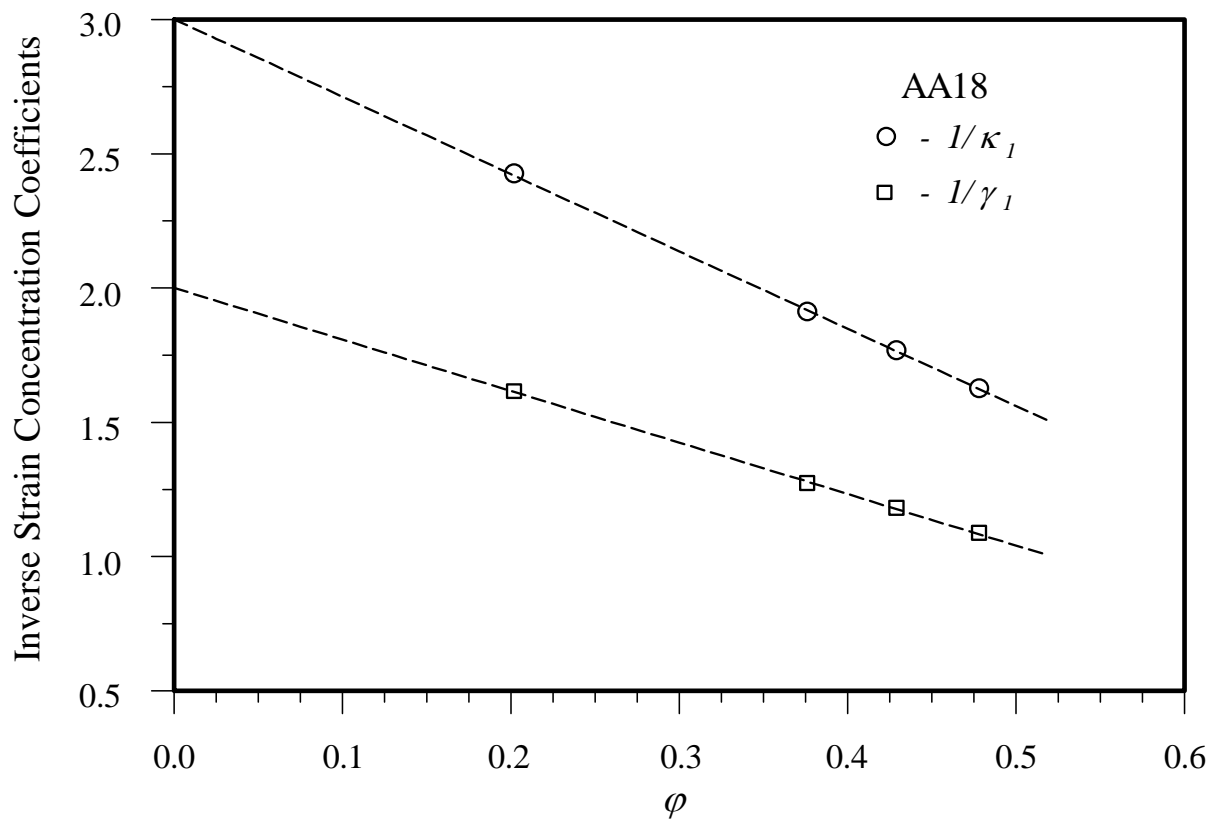

Figure 4. Values of $1 / \kappa_{1}$ and $1 / \gamma_{1}$ versus alumina volume fraction calculated for AA18 compositions. The curves shown are linear fits constrained to pass through appropriate values for each coefficient at $\varphi=0$. 


\section{Strain Concentration Factors for AA5 Compositions}

Table 6 provides a listing of the reinforcement volume fraction and strain concentration coefficients determined for the AA5 compositions examined and a plot of the inverses of these strain concentration coefficients as a function of alumina volume fraction is shown in Figure 5. A linear least squares fit, constrained to pass through the appropriate limiting values at $\varphi=0$, to the inverse values of the strain concentration coefficients as a function of the reinforcement volume fraction was again performed and is shown in Figure 5. The least square fits to these points for AA5 compositions gives $A_{A A 5}=-0.9527$ and $B_{A A 5}=-0.9589$.

Table 6. Strain concentration coefficients for AA5 compositions.

\begin{tabular}{cccccc}
\hline \hline $\begin{array}{c}\rho \\
\left(\mathrm{kg} / \mathrm{m}^{3}\right)\end{array}$ & $\begin{array}{c}\varphi \\
(\%)\end{array}$ & $\begin{array}{c}K \\
(\mathrm{GPa})\end{array}$ & $\begin{array}{c}G \\
(\mathrm{GPa})\end{array}$ & $\kappa_{1}$ & $\gamma_{1}$ \\
\hline 1765 & 20.4 & 8.42 & 2.87 & 0.413 & 0.621 \\
2391 & 43.0 & 14.0 & 6.01 & 0.567 & 0.854 \\
\hline \hline
\end{tabular}

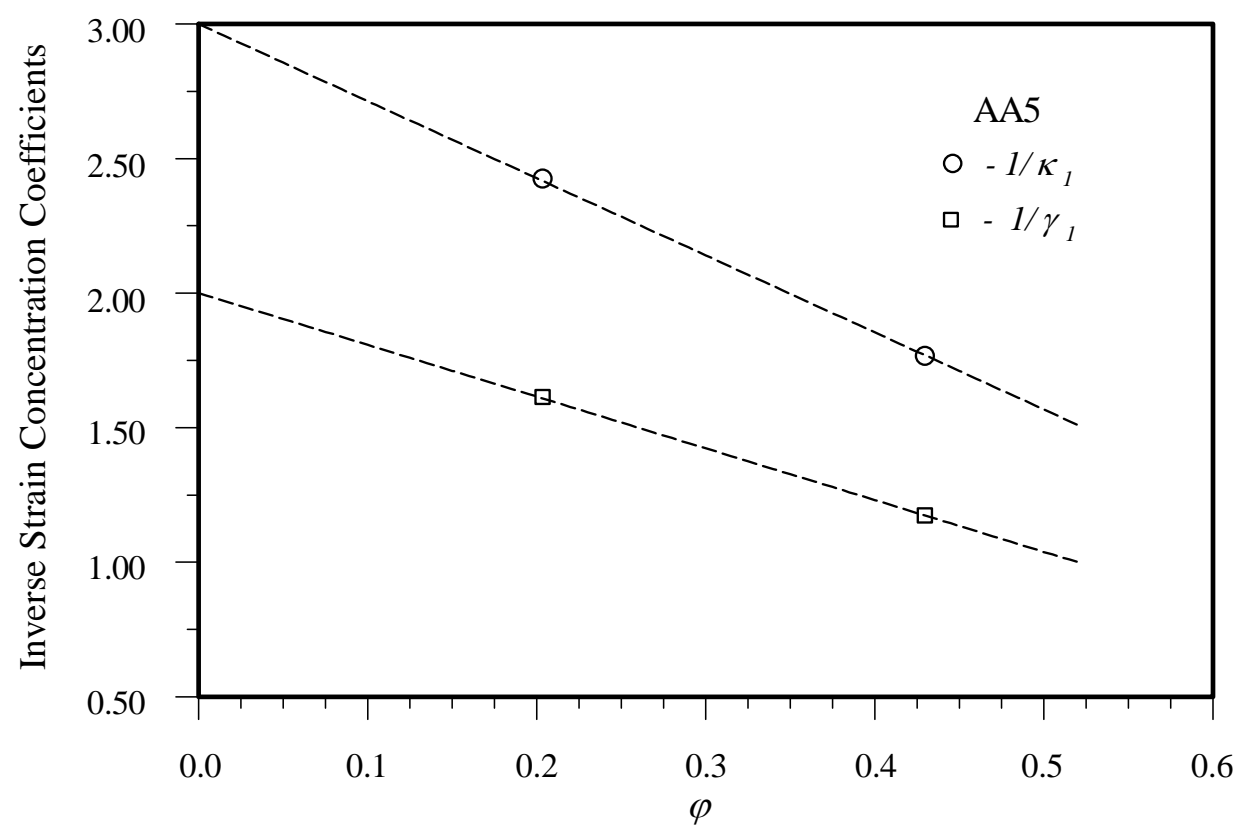

Figure 5. Values of $1 / \kappa_{1}$ and $1 / \gamma_{1}$ versus alumina volume fraction calculated for AA5 compositions. The curves shown are linear fits constrained to pass through appropriate values for each coefficient at $\varphi=0$. 


\section{E. Effect of Particle Type on Strain Concentration Coefficients}

Table 7 lists the values of the parameters $A$ and $B$ determined using the least square fitting procedure described above for each type of alumina particle. Examination of the values listed in Table 7 indicate that $A$ and $B$ are nearly equal for the alumina particle types examined.

Table 7. Composition parameters for the alumina-filled epoxies examined.

\begin{tabular}{cccc}
\hline \hline \multirow{2}{*}{$\begin{array}{c}\text { Composition } \\
\text { Parameters }\end{array}$} & T64 & AA18mina Type & AA5 \\
\cline { 2 - 4 } & -0.9545 & -0.9579 & -0.9527 \\
$B$ & -0.9564 & -0.9574 & -0.9589 \\
\hline \hline
\end{tabular}

Because of the near equality of values for $A$ and $B$ listed in Table 7 it is reasonable to conclude that the reinforcement volume fraction is the most significant factor controlling the effective elastic properties of the composite materials examined. Particle morphology and size do not appear have any significant effect on the effective elastic properties of the composite materials examined.

\section{F. An Examination of the Analytic Approximations}

It is informative to investigate the effectiveness of the three approximation methods for the strain concentration coefficients of the composite described in the previous section. The rule of mixtures gives $\kappa_{1}^{\varepsilon}=1 / 3$ and $\gamma_{1}^{\varepsilon}=1 / 2$. The results above indicate that these values for the strain concentration coefficients are only approached as the volume fraction of alumina particle reinforcement vanishes. Consequently, the rule of mixtures does not appear to be viable in estimating the effective elastic properties for epoxy reinforced with even small amounts of alumina particles. The inverse rule of mixtures appears to provide a better method for estimating the effective elastic properties. A comparison of the approximations for the strain concentration coefficients, given by (2.20), provided using the inverse rule of mixtures and (3.4) gives

$$
A^{\sigma}=\left(\frac{K_{1}}{K_{2}}\right)-1 \approx-0.9769 \text { and } B^{\sigma}=\left(\frac{G_{1}}{G_{2}}\right)-1 \approx-0.9890 \text {. }
$$

Here the superscript ' $\sigma$ ' on $A$ and $B$ indicates predictions of these parameters using the inverse rule of mixtures. These predictions for $A$ and $B$ are only slightly larger than the corresponding 
values listed in Table 7. The approximation for the strain concentration coefficients obtained assuming a dilute suspension of particles in the epoxy matrix results in

$$
\begin{aligned}
& A^{D}=a-1=\frac{\left(K_{1} / K_{2}\right)-1}{1+\left(4 G_{1} / 3 K_{2}\right)} \approx-0.9683 \\
& B^{D}=b-1=\frac{\left(G_{1} / G_{2}\right)-1}{1+\left[\left(9 K_{1}+8 G_{1}\right) / 6\left(K_{1}+2 G_{1}\right)\right]\left(G_{1} / G_{2}\right)} \approx-0.9762 .
\end{aligned}
$$

Here the superscript ' $D$ ' on $A$ and $B$ indicates predictions of these parameters using the dilute suspension assumption rule. These predictions for $A$ and $B$ are somewhat lower than those obtained using the inverse rule of mixtures and again only slightly larger than the corresponding values listed in Table 7. It is interesting to note that the inverse rule of mixtures and dilute suspension approximation predict larger differences in the parameters $A$ and $B$ than found in the analysis of actual measurements.

Figure 6 illustrates the dependence of the strain concentration coefficient $\gamma_{1}$ on the reinforcement volume fraction of alumina calculated using (3.3) with values of $B$ from the various estimates and the data fit, from Table 7, for the T64 compositions. Curves illustrating the

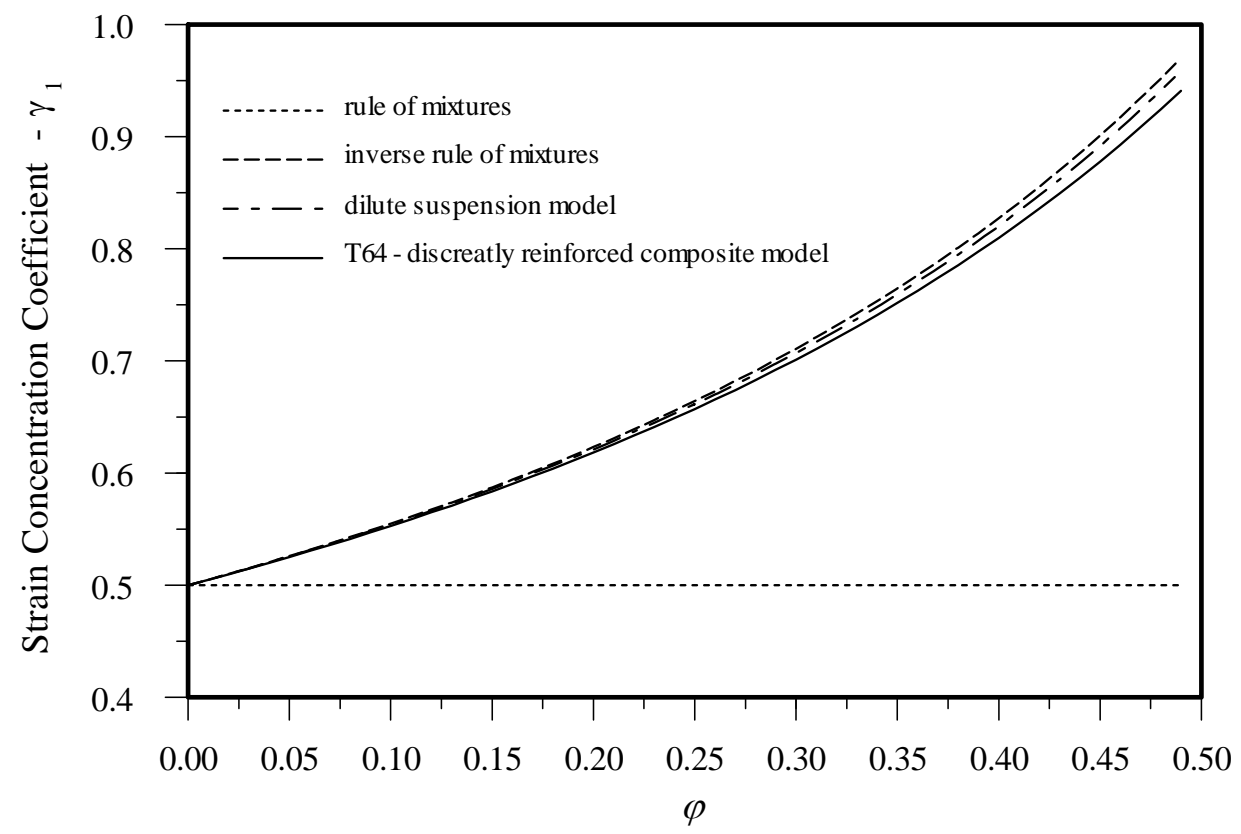

Figure 6. Dependence of $\gamma_{1}$ on alumina reinforcement volume fraction using values of $B$ determined by the estimation methods discussed and the data fit for compositions using T64 alumina. 
dependence of $\gamma_{1}$ on reinforcement volume fraction for the values of $B$ in Table 7 corresponding the AA18 and AA5 compositions are not shown since they lie within a line width of the curve, slightly below and above respectively, shown using the $B$ value for T64 compositions. Corresponding plots of the dependence of $\kappa_{1}$ on reinforcement volume fraction are similar to those shown in Figure 5 but even more tightly grouped.

Equations (2.16) and (3.4) can be used to calculate the effective bulk and shear moduli for the composite material at a particular reinforcement volume fraction using the model parameters listed in Tables 3 and 7. Figures 7 and 8 show the calculated dependence of the effective bulk and shear moduli on reinforcement volume fraction using the model parameters obtained for the T64, AA18, and AA5 compositions as well using estimates for $A$ and $B$ provided by the rule of mixtures, inverse rule of mixtures, and dilute suspension model. Values of effective bulk and shear modulus obtained from density and sound speed measurements on the various composite samples listed in Table 1 are shown for comparison.

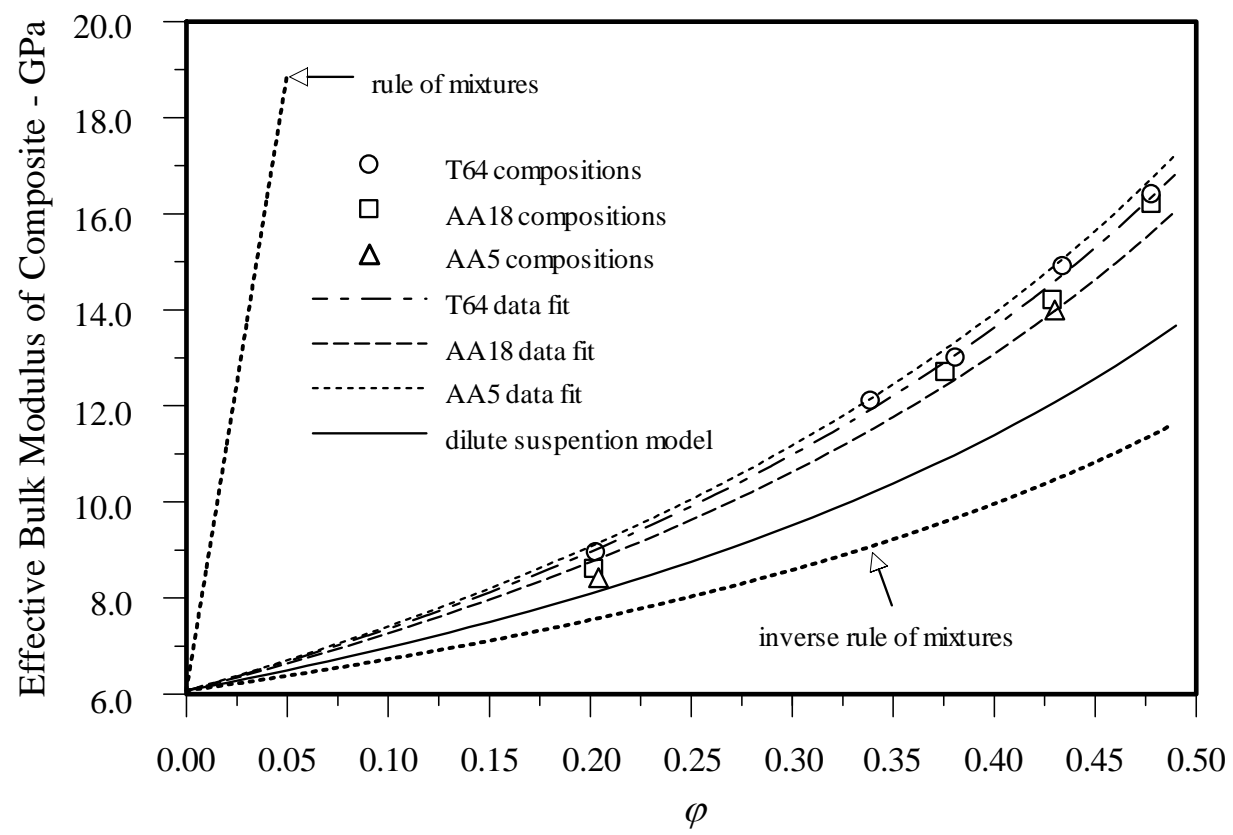

Figure 7. Graphical illustrations of the predicted dependence of the effective bulk modulus on reinforcement volume fraction for alumina filled epoxy. 


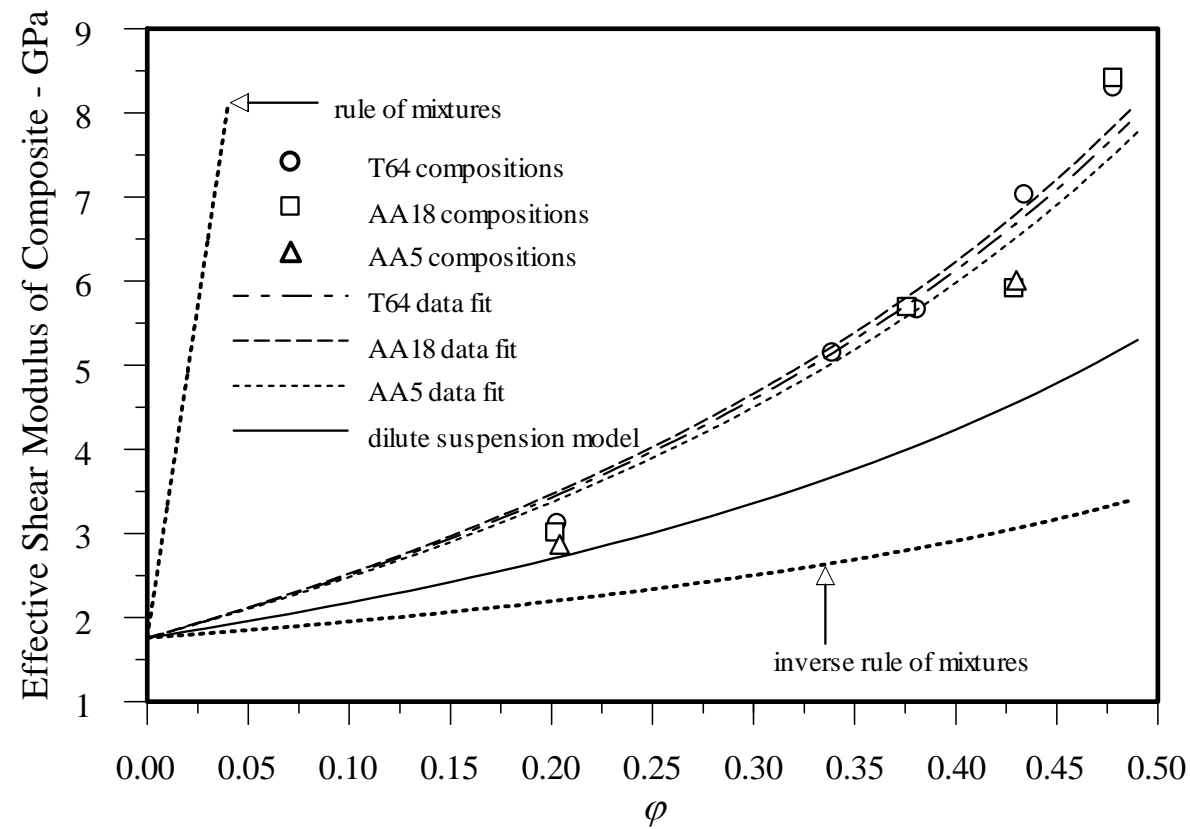

Figure 8. Graphical illustrations of the predicted dependence of the effective shear modulus on reinforcement volume fraction for alumina filled epoxy.

Examination of the Figures 7 and 8 indicate that none of the predictive methods for estimating the parameters $A$ and $B$ provide reliable estimates for the effective elastic moduli over the range of reinforcement volume fraction examined. Even though the values of $A$ and $B$ for the predictive methods were only slightly larger than values deduced from experimental measurements, the large discrepancy in elastic moduli for the epoxy and alumina greatly magnifies the small differences in values of $\kappa_{1}$ and $\gamma_{1}$ when using (2.16).

The large values of elastic moduli for the alumina also magnify the uncertainty in calculating values for $\kappa_{1}$ and $\gamma_{1}$ when using (2.17). Since the effective elastic moduli are much smaller than the elastic moduli for the alumina reinforcement, the errors in the effective elastic moduli reflecting uncertainties in the measured values of density and sound speeds for the composite samples are significantly reduced. In particular, since the sample-to-sample variations in density, longitudinal sound speed, and shear sound speed were $\pm 5 \mathrm{~kg} / \mathrm{m}^{3}, \pm 19 \mathrm{~m} / \mathrm{s}$, and $\pm 9 \mathrm{~m} / \mathrm{s}$, respectively, the uncertainty in the calculated values for the effective elastic moduli are on the 
order of $\pm 1 \mathrm{GPa}$. Consequently, while evident discrepancy between the predicted and measured values for the effective elastic moduli of the compositions can be seen in Figures 7 and 8, the predicted values for the effective moduli obtained by fitting the strain concentration coefficients are much closer to the measurements than the predictions obtained using analytic approximations for the range of alumina volume fraction investigated. 


\section{The Response of Alumina-Filled Epoxy to Large Compression}

It is of interest to examine connections between the linear elastic response model developed above and response models applicable to situations involving large compression, such as are

produced by the passage of a shock-wave through the composite in plate impact experiments. ${ }^{1,2}$ Hydrostatic and uniaxial plane-strain responses of alumina-filled epoxy to large compression are investigated in this section. The response model described below has a similar structure to the linear elastic response model characterized above, and results from the linear elastic response model are used to characterize the large compression response models.

\section{A. Preliminaries}

The volume fraction of alumina in the composite was regarded as fixed in the linear elastic response model. Because of the very large stiffness of the alumina particles relative to the epoxy matrix, it is expected that large compression of the composite will result in a significant increase in the volume fraction of alumina from its initial value in the uncompressed stress-free state. Consequently, a response model valid for large compression will need to allow for changes in the volume fraction of the alumina particles as the composite is compressed.

Consider a representative volume of the composite of fixed mass $M$ under a uniform compression and let $M_{1}$ and $M_{2}$ be the masses of the epoxy and alumina, respectively, in the mixture. The mass fractions of epoxy and alumina in the composite are then given by $m_{1}=M_{1} / M$ and $m_{2}=M_{2} / M$, respectively. In the absence of voids and chemical reactions these two mass fractions remain constant for any deformation and sum to unity. Recalling the definition of the volume fractions of epoxy and alumina gives

$$
(1-\varphi)=\left(1-m_{2}\right) \frac{\tilde{\rho}}{\tilde{\rho}_{1}} \quad \text { and } \quad \varphi=m_{2} \frac{\tilde{\rho}}{\tilde{\rho}_{2}} .
$$

Since $m_{2}$ remains constant, we can write

$$
\frac{\tilde{\rho}_{1}}{\tilde{\rho}_{10}}=\frac{\left(1-\varphi_{0}\right)}{(1-\varphi)} \frac{\tilde{\rho}}{\tilde{\rho}_{0}} \quad \text { and } \quad \frac{\tilde{\rho}_{2}}{\tilde{\rho}_{20}}=\frac{\varphi_{0}}{\varphi} \frac{\tilde{\rho}}{\tilde{\rho}_{0}}
$$

where the added subscript ' 0 ' indicates the value of a quantity in the stress-free reference state. Equation (4.2) provides relations between the average densities of the epoxy and alumina given 
the average density of the composite and the volume fraction of alumina. For small deformations we have

$$
\frac{\tilde{\rho}_{01}}{\tilde{\rho}_{1}} \approx 1+\tilde{e}_{k k}^{(1)}, \quad \frac{\tilde{\rho}_{02}}{\tilde{\rho}_{2}} \approx 1+\tilde{e}_{k k}^{(2)}, \quad \text { and } \quad \frac{\tilde{\rho}_{0}}{\tilde{\rho}} \approx 1+\tilde{e}_{k k} .
$$

Consequently, (4.2) yields

$$
\tilde{e}_{k k}^{(1)} \approx \frac{\left[\left(\varphi_{0}-\varphi\right)+(1-\varphi) \tilde{e}_{k k}\right]}{\left(1-\varphi_{0}\right)} \text { and } \tilde{e}_{k k}^{(2)} \approx \frac{\left[\left(\varphi-\varphi_{0}\right)+\varphi \tilde{e}_{k k}\right]}{\varphi_{0}},
$$

which gives

$$
\left(1-\varphi_{0}\right) \tilde{e}_{k k}^{(1)}+\varphi_{0} \tilde{e}_{k k}^{(2)} \approx \tilde{e}_{k k}
$$

Consequently, (4.2) is compatible with the linear elastic theory for small deformations.

It is convenient to use the alumina distention $\alpha$, which is the inverse of the alumina volume fraction, in the following discussion. We have

$$
\alpha=\frac{1}{\varphi}=\frac{\left(\tilde{\rho}_{2}-\tilde{\rho}_{1}\right)}{\left(\tilde{\rho}-\tilde{\rho}_{1}\right)}>1 .
$$

The alumina distention decreases as the volume fraction increases. Clearly, the alumina distension is an alternate representation of the alumina volume fraction for the composite.

\section{B. Hydrostatic Compression}

In the special case of hydrostatic compression the diagonal terms of the stress and strain tensors are all equal and the off-diagonal terms vanish. The average stresses describing the composite then take the form

$$
\begin{aligned}
& \tilde{\sigma}_{11}=\tilde{\sigma}_{22}=\tilde{\sigma}_{33}=-\tilde{P}, \quad \tilde{\sigma}_{12}=\tilde{\sigma}_{23}=\tilde{\sigma}_{13}=0 \\
& \tilde{\sigma}_{11}^{(1)}=\tilde{\sigma}_{22}^{(1)}=\tilde{\sigma}_{33}^{(1)}=-\tilde{P}^{(1)}, \quad \tilde{\sigma}_{12}^{(1)}=\tilde{\sigma}_{23}^{(1)}=\tilde{\sigma}_{13}^{(1)}=0 \\
& \tilde{\sigma}_{11}^{(2)}=\tilde{\sigma}_{22}^{(2)}=\tilde{\sigma}_{33}^{(2)}=-\tilde{P}^{(2)}, \quad \tilde{\sigma}_{12}^{(2)}=\tilde{\sigma}_{23}^{(2)}=\tilde{\sigma}_{13}^{(2)}=0 \text {. }
\end{aligned}
$$

Here $\tilde{P}, \tilde{P}^{(1)}$, and $\tilde{P}^{(2)}$ are, respectively, the average pressures in the composite, epoxy, and alumina. It follows from (2.1), (4.6), and (4.7) that these pressures are related to each other by

$$
\alpha\left[\tilde{P}-\tilde{P}^{(1)}\right]=\tilde{P}^{(2)}-\tilde{P}^{(1)} .
$$

It is convenient to use specific volumes $(v=1 / \rho)$ in the following derivations. Equation (4.2) becomes 


$$
\tilde{v}_{1}=\tilde{v}_{10} \frac{(\alpha-1)}{\left(\alpha_{0}-1\right)}\left(\frac{\alpha_{0}}{\alpha}\right)\left(\frac{\tilde{v}}{\tilde{v}_{0}}\right) \text { and } \tilde{v}_{2}=\tilde{v}_{20}\left(\frac{\alpha_{0}}{\alpha}\right)\left(\frac{\tilde{v}}{\tilde{v}_{0}}\right) \text {. }
$$

Since the pressures in the epoxy and alumina will depend on the change in the average densities from the pressure-free reference configuration, the following relations are used for the pressures in the epoxy and the alumina:

$$
\begin{aligned}
& \tilde{P}^{(1)}=\tilde{P}^{(1)}\left(\tilde{v}_{1}\right)=\frac{K_{1}\left[1-\left(\tilde{v}_{1} / \tilde{v}_{10}\right)\right]}{\left\{1-S\left[1-\left(\tilde{v}_{1} / \tilde{v}_{10}\right)\right]\right\}^{2}} \\
& \tilde{P}^{(2)}=\tilde{P}^{(2)}\left(\tilde{v}_{2}\right)=K_{2}\left[1-\left(\tilde{v}_{2} / \tilde{v}_{20}\right)\right]
\end{aligned}
$$

The pressure in the epoxy is allowed a nonlinear dependence on compression while the pressure in the alumina remains linear in compression due to its large stiffness in comparison to epoxy.

Equations (4.2), (4.8), (4.9), and (4.10) need to be augmented by one additional relation to form a response model for the hydrostatic compression of the composite. It does not seem reasonable to expect the linear transformations between the volumetric strains in the composite and component materials to hold for large compression. A related approach is used here by defining an intermediate variable $\tilde{P}^{s}=\tilde{P}-\tilde{P}^{(1)}$, called the structural pressure, and assuming that the structural pressure is a function of the alumina distention, i.e.,

$$
\tilde{P}^{s}=\tilde{P}^{s}(\alpha) \text { where } \tilde{P}=\tilde{P}^{s}+\tilde{P}^{(1)} \text {. }
$$

This equation is presumed to reflect equilibrium between the pressure in the composite and the deformation as specified by the alumina distention. The strain concentration coefficients played a similar role in the linear elastic theory where it was assumed that stress equilibrium controlled the linear transformation between average strains in the composite and its components.

The specific instance in which (4.12) can be written as

$$
\alpha(\alpha-1) \tilde{P}^{s}=k\left(\alpha-\alpha_{0}\right)
$$

where $k$ is a material constant having units of pressure, is examined. Use of (4.13) is motivated as follows. Presume that the aggregate of alumina particles in the composite supports a linked network that resists deformation with forces being transmitted between the particles through the epoxy. In a porous solid, the term $\tilde{P}^{s}$ occurring in (4.12) is just the mean stress in the matrix and (4.13) is a special form relating the average mean stress and distention of the solid matrix material obtained from the linear elastic response of a hollow sphere ${ }^{15}$ loaded by a hydrostatic 
pressure on its outer surface while its inner surface remains free of stress. It is clear that (4.13) would not be altered if a uniform pressure was added to the existing pressures at the inner and outer surfaces and the solid material regarded as incompressible. Since the alumina is much stiffer than the epoxy, it seems feasible to regard the suspension of alumina as an effective solid matrix with the epoxy forming an effective pore space. Equation (4.13) then gives a relation between the alumina distention and structural pressure $\tilde{P}_{S}$, which plays the role of the effective mean stress for the network of forces between the particles. The parameter $k$ appearing in (4.13) plays a role similar to that of $\kappa_{1}$ in reflecting the effect of microstructure on the equilibrium structural pressure as the distention changes from its initial value.

It is useful to examine the average isothermal bulk modulus, $\tilde{\beta}$, of the effective solid, defined by the expression

$$
\tilde{\beta}=-\tilde{v} \frac{d \tilde{P}}{d \tilde{v}},
$$

to help in characterizing the large compression response model. Using (4.14) with (4.12) and (4.9) gives

$$
\tilde{\beta}=\tilde{\beta}_{1}-\tilde{v}\left\{\left(\frac{d \tilde{P}_{S}}{d \alpha}\right)-\frac{\tilde{\beta}_{1}}{\alpha(\alpha-1)}\right\}\left(\frac{d \alpha}{d \tilde{v}}\right) .
$$

Combining (4.8) and (4.12) with the constraint of (4.9) yields

$$
\left(\frac{d \alpha}{d \tilde{v}}\right)=-\frac{\alpha(\alpha-1)\left[\tilde{\beta}_{2}-\tilde{\beta}_{1}\right]}{\tilde{v}\left\{\alpha(\alpha-1)\left[\tilde{P}^{s}+\alpha\left(\frac{d \tilde{P}^{s}}{d \alpha}\right)\right]-\tilde{\beta}_{1}-(\alpha-1) \tilde{\beta}_{2}\right\}} .
$$

Substituting (4.16) into (4.15) then gives

$$
\tilde{\beta}=\tilde{\beta}_{1}+\frac{\left[\alpha(\alpha-1)\left(\frac{d \tilde{P}_{S}}{d \alpha}\right)-\tilde{\beta}_{1}\right]\left[\tilde{\beta}_{2}-\tilde{\beta}_{1}\right]}{\left\{\alpha(\alpha-1)\left[\tilde{P}^{S}+\alpha\left(\frac{d \tilde{P}_{S}}{d \alpha}\right)\right]-\tilde{\beta}_{1}-(\alpha-1) \tilde{\beta}_{2}\right\}} .
$$

The reference state of the composite is characterized with $\alpha=\alpha_{0}, \tilde{P}^{S}=0, \tilde{\beta}=K, \tilde{\beta}_{1}=K_{1}$, and $\tilde{\beta}_{2}=K_{2}$, so that (4.17) yields 


$$
K=K_{1}+\frac{\left[\alpha_{0}\left(\alpha_{0}-1\right)\left(\frac{d \tilde{P}_{S}}{d \alpha}\right)_{\alpha=\alpha_{0}}-K_{1}\right]\left[K_{2}-K_{1}\right]}{\left\{\alpha_{0}^{2}\left(\alpha_{0}-1\right)\left(\frac{d \tilde{P}_{S}}{d \alpha}\right)_{\alpha=\alpha_{0}}-K_{1}-\left(\alpha_{0}-1\right) K_{2}\right\}} .
$$

Solving for the initial rate of change in $\tilde{P}^{s}$ with respect to the alumina distention gives

$$
\left(\frac{d \tilde{P}_{S}}{d \alpha}\right)_{\alpha=\alpha_{0}}=\frac{\left(\alpha_{0}-1\right) K_{2}-\left[K_{1}+\left(\alpha_{0}-1\right) K_{2}\right]\left[\left(K-K_{2}\right) /\left(K_{1}-K_{2}\right)\right]}{\alpha_{0}\left(\alpha_{0}-1\right)\left\{\left(\alpha_{0}-1\right)-\alpha_{0}\left[\left(K-K_{2}\right) /\left(K_{1}-K_{2}\right)\right]\right\}} .
$$

Equations (3.4) and (2.17) combine to yield

$$
\frac{\left(K-K_{2}\right)}{\left(K_{1}-K_{2}\right)}=3\left(1-\varphi_{0}\right) \kappa_{1}=\frac{\left(1-\varphi_{0}\right)}{\left(1+A \varphi_{0}\right)}=\frac{\left(\alpha_{0}-1\right)}{\left(\alpha_{0}+A\right)},
$$

which can be substituted into (4.18) to provide

$$
\left(\frac{d \tilde{P}_{S}}{d \alpha}\right)_{\alpha=\alpha_{0}}=\frac{(1+A) K_{2}-K_{1}}{\alpha_{0}\left(\alpha_{0}-1\right) A} .
$$

Consequently, the large compression model recovers the response predicted by the linear elastic theory when

$$
k=\frac{(1+A) K_{2}-K_{1}}{A} .
$$

Equation (4.21) connects the large compression response model to the linear elastic response model by relating the parameters $A$ and $k$.

The calculation of the hydrostat predicted using the large compression response model is now considered. Let the volumetric compression $\tilde{\varepsilon}_{V}$ be fixed. Assuming the volumetric compression is reckoned as positive, the corresponding specific volume of the composite is

$$
\tilde{v}=\tilde{v}_{0}\left(1-\tilde{\varepsilon}_{V}\right) .
$$

If it is assumed that the alumina distention is known, then (4.9), (4.10), and (4.11) give the appropriate values of $\tilde{P}^{(1)}$ and $\tilde{P}^{(2)}$ for the composite. In order to determine $\alpha$, the nonlinear algebraic equation obtained by combining (4.8) and (4.13),

$$
k\left(\alpha-\alpha_{0}\right)-(\alpha-1)\left[\tilde{P}^{(2)}(\alpha)-\tilde{P}^{(1)}(\alpha)\right]=0,
$$

is solved using (4.21) with (4.9) to constrain the compressions so that (4.10) and (4.11) give the average pressures in the epoxy and alumina corresponding a particular value of $\alpha$. 
Figure 9 illustrates predictions for the dependence of the hydrostatic pressure on volume strain for a composite with an initial alumina distention $\alpha_{0} \approx 2.304$, which corresponds to an initial volume fraction $\varphi_{0} \approx 0.434$. The curve showing the lowest prediction for the hydrostatic pressure results from using the linear elastic response model. The three curves giving higher pressures result from using the large compression model developed with three different values of $S$ for the nonlinearity of the epoxy. The curve in Figure 9 labeled $S=0$ corresponds to an epoxy response that is linear in volumetric compression of the epoxy. The curves in Figure 9 labeled with $S=1$ and 2 show the effect of the nonlinear response of the epoxy on the hydrostatic pressure. Since only small differences in the alumina distention were found for the three predictions using the large compression model, it is concluded that the nonlinearity in the hydrostatic pressure is primarily due to the nonlinearity of the epoxy.

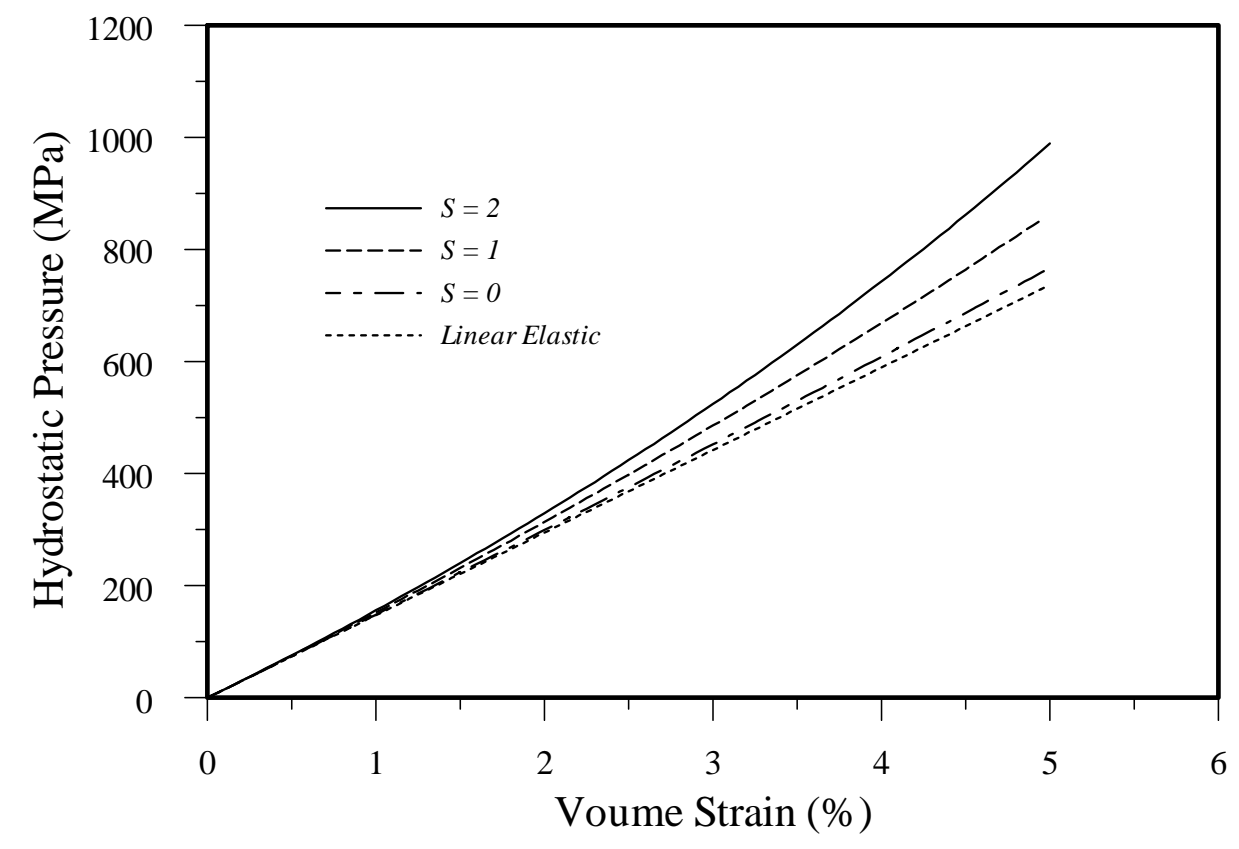

Figure 9. Predicted hydrostatic response for alumina-filled epoxy with an initial alumina volume fraction of $43.4 \%$. Predictions are shown for the linear and large compression models. Increasing values of $S$ correspond to an increasing nonlinear dependence of pressure on compression for the epoxy. 


\section{Uniaxial Plane- Strain Compression}

The special case of uniaxial plane-strain compression occurs in plate impact experiments used to characterize the response of the composite under shock-wave compression. ${ }^{1,2,4}$ In this case, the average motion of the composite can be regarded as one-dimensional and the only non-vanishing component of the average strain tensor in the composite is the normal component on the surface normal to the direction of shock-wave propagation. As a consequence, the components of average shear stresses in the composite will vanish producing an average principal compressive stress state (compressive stresses will be reckoned as positive in this discussion). The largest average principal stress is the normal compressive stress acting on the plane defined by the direction of shock-wave propagation. The other two average principal stresses are two equal normal compressive stresses acting on planes perpendicular to the direction of shock-wave propagation. If the direction of shock-wave propagation is along the $x_{1}$ - direction, these average strains and stresses in the composite can be written

$$
\begin{array}{r}
\tilde{\varepsilon}_{11}=\tilde{\varepsilon}_{C}=1-\left(\tilde{v} / \tilde{v}_{0}\right), \quad \tilde{\varepsilon}_{22}=\tilde{\varepsilon}_{33}=\tilde{\varepsilon}_{12}=\tilde{\varepsilon}_{23}=\tilde{\varepsilon}_{13}=0 \\
\tilde{\sigma}_{11}=\tilde{\sigma}_{C}, \quad \tilde{\sigma}_{22}=\tilde{\sigma}_{33}=\tilde{\sigma}_{L}, \quad \tilde{\sigma}_{12}=\tilde{\sigma}_{23}=\tilde{\sigma}_{13}=0
\end{array}
$$

where $\tilde{\varepsilon}_{C}$ is the average compressive strain, $\tilde{\sigma}_{C}$ is the compressive stress, and $\tilde{\sigma}_{L}$ is the lateral confining stress.

It is useful to work with the strain, $\tilde{e}_{i j}$, and stress, $\tilde{s}_{i j}$, deviation tensors defined by

$$
\begin{aligned}
& \tilde{e}_{i j}=\tilde{\varepsilon}_{i j}-\frac{1}{3} \tilde{\varepsilon}_{k k} \delta_{i j} \\
& \tilde{s}_{i j}=\tilde{\sigma}_{i j}-\frac{1}{3} \tilde{\sigma}_{k k} \delta_{i j} .
\end{aligned}
$$

For plane-strain compression $\tilde{\varepsilon}_{k k}=\tilde{\varepsilon}_{C}, \tilde{\sigma}_{k k}=\tilde{\sigma}_{C}+2 \tilde{\sigma}_{L}$, and the average components of the strain and stress deviation tensors are

$$
\begin{array}{cc}
\tilde{e}_{11}=\frac{2 \tilde{\varepsilon}_{C}}{3}, \quad \tilde{e}_{22}=\tilde{e}_{33}=-\frac{\tilde{\varepsilon}_{C}}{3}, \quad \tilde{e}_{12}=\tilde{e}_{23}=\tilde{e}_{13}=0 \\
\tilde{s}_{11}=\frac{4 \tilde{\tau}}{3}, \quad \tilde{s}_{22}=\tilde{s}_{33}=-\frac{2 \tilde{\tau}}{3}, \quad \tilde{s}_{12}=\tilde{s}_{23}=\tilde{s}_{13}=0
\end{array}
$$

where $\tilde{\tau}=\left(\tilde{\sigma}_{C}-\tilde{\sigma}_{L}\right) / 2$.

The average linear elastic response of the composite can be written in terms of the components of the stress and strain deviation tensors as 


$$
\begin{gathered}
\frac{1}{3} \tilde{\sigma}_{k k}=\tilde{\sigma}_{M}=K \tilde{\varepsilon}_{k k} \\
\tilde{s}_{i j}=2 G \tilde{e}_{i j}
\end{gathered}
$$

where $\tilde{\sigma}_{M}$ is the average mean stress in the material. For plane-strain compression (4.29) and (4.30) become

$$
\begin{gathered}
\tilde{\sigma}_{M}=K \tilde{\varepsilon}_{C} \\
\tilde{\tau}=G \tilde{e}_{C} .
\end{gathered}
$$

We note that $\tilde{\sigma}_{M}$ and $\tilde{\tau}$ can be expressed in terms of invariants of the average stress tensor and that $\tilde{\varepsilon}_{C}$ is the first invariant of the average strain tensor. The two non-vanishing components of the average stress can be written in terms of $\tilde{\sigma}_{M}$ and $\tilde{\tau}$ giving

$$
\begin{aligned}
& \tilde{\sigma}_{C}=\tilde{\sigma}_{M}+\frac{4}{3} \tilde{\tau} \\
& \tilde{\sigma}_{L}=\tilde{\sigma}_{M}-\frac{2}{3} \tilde{\tau} .
\end{aligned}
$$

A response model giving equilibrium values for $\tilde{\sigma}_{C}$ in terms of $\tilde{\varepsilon}_{C}$ for large compressions due plane shock-waves is sought. Equation (4.33) can be decomposed into the contributions from the average normal stress in the epoxy and alumina as

$$
\alpha\left(\tilde{\sigma}_{C}-\tilde{\sigma}_{C}^{(1)}\right)=\tilde{\sigma}_{C}^{(2)}-\tilde{\sigma}_{C}^{(1)} .
$$

The structural normal stress is defined as

$$
\tilde{\sigma}_{C}^{S}=\tilde{\sigma}_{C}-\tilde{\sigma}_{C}^{(1)}
$$

It is now postulated that the distention of alumina can be related to the invariants of the structural stress in the composite, i.e.,

$$
\alpha=\alpha\left(\tilde{\sigma}_{M}^{S}, \tilde{\tau}^{S}\right)
$$

Since (4.37) should reduce to (4.13) when $\tilde{\tau}^{S}$ is zero, the following form is assumed to hold for the case of plane-strain compression

$$
\left(\alpha-\alpha_{0}\right)=\alpha(\alpha-1)\left[\left(\frac{\tilde{\sigma}_{M}^{S}}{k}\right)+\left(\frac{\hat{\tau}^{S}}{g}\right)\right] .
$$

The parameter $k$ appearing in (4.38) has already been determined. The parameter $g$ is selected to give the same shear stiffness in the composite as the linear response theory in the limit of 
small deformation, hence parameters for the linear elastic and large compression response are related by

$$
g=\frac{(1+B) G_{2}-G_{1}}{B}
$$

The responses of the epoxy and alumina for large plane-strain compression are assumed to be governed by

$$
\begin{aligned}
& \tilde{v}_{1}=\tilde{v}_{10} \frac{(\alpha-1)}{\left(\alpha_{0}-1\right)}\left(\frac{\alpha_{0}}{\alpha}\right)\left(\frac{\tilde{v}}{\tilde{v}_{0}}\right) \quad \text { and } \quad \tilde{v}_{2}=\tilde{v}_{20}\left(\frac{\alpha_{0}}{\alpha}\right)\left(\frac{\tilde{v}}{\tilde{v}_{0}}\right) \\
& \tilde{\sigma}_{M}^{(1)}=\frac{K_{1}\left[1-\left(\tilde{v}_{1} / \tilde{v}_{10}\right)\right]}{\left\{1-S\left[1-\left(\tilde{v}_{1} / \tilde{v}_{10}\right)\right]\right\}^{2}} \\
& \tilde{\sigma}_{M}^{(2)}=K_{2}\left[1-\left(\tilde{v}_{2} / \tilde{v}_{20}\right)\right] \\
& \tilde{\tau}^{(1)}=\left\{\begin{array}{cc}
G_{1}\left[1-\left(\tilde{v}_{1} / \tilde{v}_{10}\right)\right] & \tilde{v}_{1}^{*}<\tilde{v}_{1} \leq \tilde{v}_{10} \\
Y_{1} & \tilde{v}_{1} \leq \tilde{v}_{1}^{*}
\end{array}\right. \\
& \tilde{\tau}^{(2)}=G_{2}\left[1-\left(\tilde{v}_{2} / \tilde{v}_{20}\right)\right]
\end{aligned}
$$

where $Y_{1}$ is the yield stress for the epoxy and $\tilde{v}_{1}^{*}=\tilde{v}_{10}\left[1-\left(Y_{1} / G_{1}\right)\right]$ is the average specific volume in the epoxy needed to produce an average shear stress equal to the yield stress. Equation (4.40) is the constraint on average specific volumes of the composite, epoxy, and alumina resulting from the mass fraction of alumina and epoxy being constant. Equations (4.41) and (4.42) are used to give the mean stress in the epoxy and alumina, respectively, and are taken to be the same as the relations between hydrostatic pressure and volumetric strain since the composite, epoxy, and alumina are assumed to be isotropic. Finally, the average shear stress invariants in the epoxy and alumina are assumed proportional to the corresponding shear strain invariant, and the epoxy is assumed to yield when the shear stress reaches a critical value $Y_{1}$. It is assumed the shear deformation of the alumina remains elastic for the levels of compression of interest in this study.

Equations (4.38) through (4.44) specify the plane-strain large compression response model for alumina-filled epoxy, and can now be used to predict the average stress $\tilde{\sigma}_{C}$ in a composite 
composition for given values of $\tilde{\varepsilon}_{C}$ and initial alumina distention $\alpha_{0}$. The procedure is similar to the one described for the hydrostatic compression response with the value of $\alpha$ obtained from

$$
\left(\alpha-\alpha_{0}\right)-(\alpha-1)\left\{\frac{\left[\tilde{\sigma}_{M}^{(2)}(\alpha)-\tilde{\sigma}_{M}^{(1)}(\alpha)\right]}{k}+\frac{\left[\tilde{\tau}^{(2)}(\alpha)-\tilde{\tau}^{(1)}(\alpha)\right]}{g}\right\}=0 .
$$

Figure 10 illustrates the predicted average normal stresses in the composite for large planestrain compression for a composite having an initial distention $\alpha_{0} \approx 2.33$ (corresponding to $\left.\varphi_{0} \approx 0.43\right)$. Composites formed by suspending T64 alumina in epoxy are expected to have $k \approx-6.18 \mathrm{GPa}$ and $g \approx-5.45 \mathrm{GPa}$. Also, a yield stress of $0.2 \mathrm{GPa}$ was assumed for the epoxy. The average compressive normal stress components shown in Figure 10 correspond to a given average compressive strain predicted by the large compression model. As can be seen, the average compressive stress in the composite is between the average compressive stresses in the epoxy and alumina. A small change in the slopes of the average normal stresses due to the small yield strength of the epoxy occurs below an average compressive strain of 0.01 .

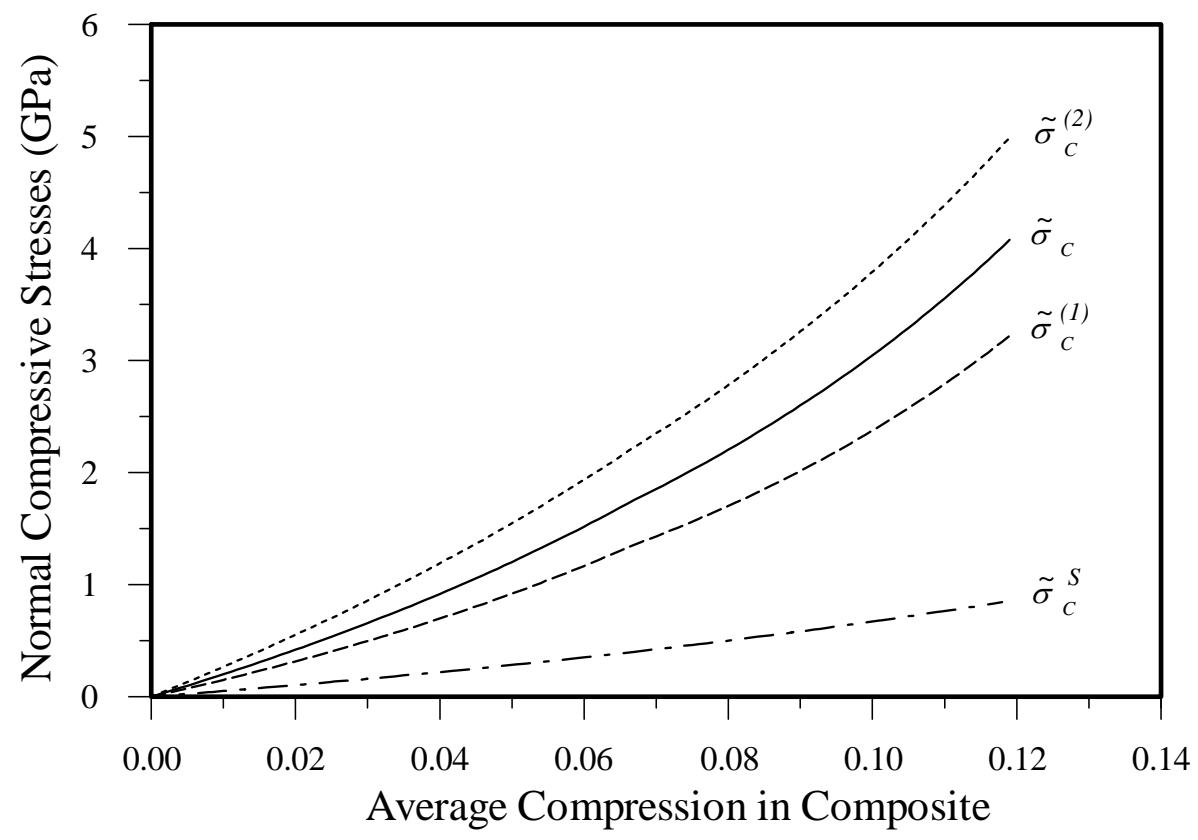

Figure 10. Average normal stresses predicted for large plane-strain compression of an alumina-filled epoxy having an initial alumina volume fraction of $43 \%$. 


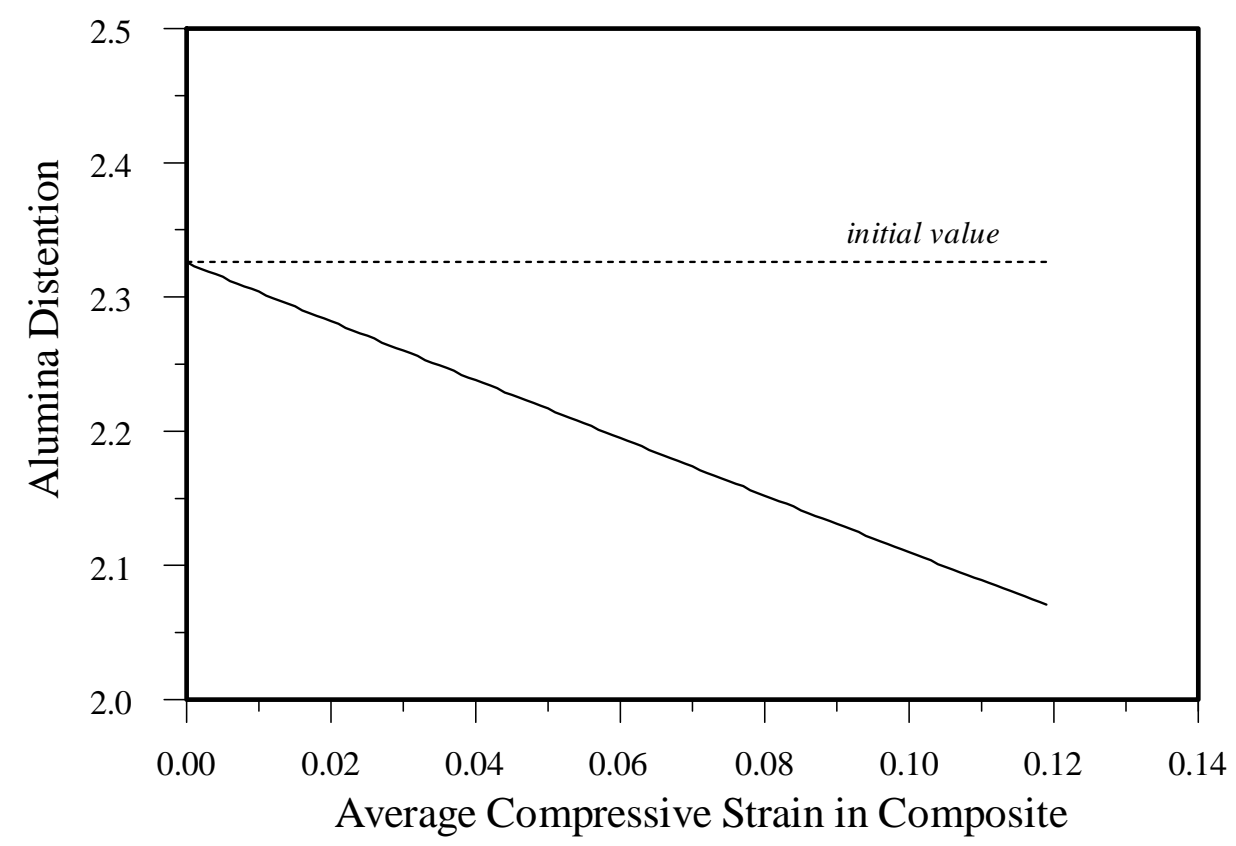

Figure 11. Alumina distention predicted for the large plane-strain compression of an alumina-filled epoxy with an initial alumina volume fraction of $43 \%$.

Figure 11 shows the change in alumina distention as a function of average compressive strain in the composite. A decrease in the alumina distention as the average compressive strain in the composite increases is seen as expected. The volume fraction of alumina is seen to increase from an initial value of $\sim 43 \%$ to a value over $\sim 48 \%$ when the compressive strain is $\sim 0.12$.

A comparison of the plane-strain compression response model predictions with measured responses on samples of alumina-filled epoxy is shown in Figure 12 for several compositions containing different initial volume fraction of T64 alumina. The measured responses correspond to the normal stress equilibrium Hugoniot states generated in plate impact experiments on the alumina-filled epoxy compositions. The normal stress predictions were calculated for initial alumina volume fractions of $0.5,20,34,38,43$, and $48 \%$ assuming the epoxy response was characterized using the parameters reported above with $S=1.78$.

The open squares in Figure 12 correspond to equilibrium Hugoniot states determined for each composition by impacting a sample of the composition with a plate of the same composition and maintaining a nearly constant impact speed for each test. Examination of Figure 12 shows that the stresses predicted by the model are reasonably close to the measured values. Numerous ${ }^{1,2,4}$ 


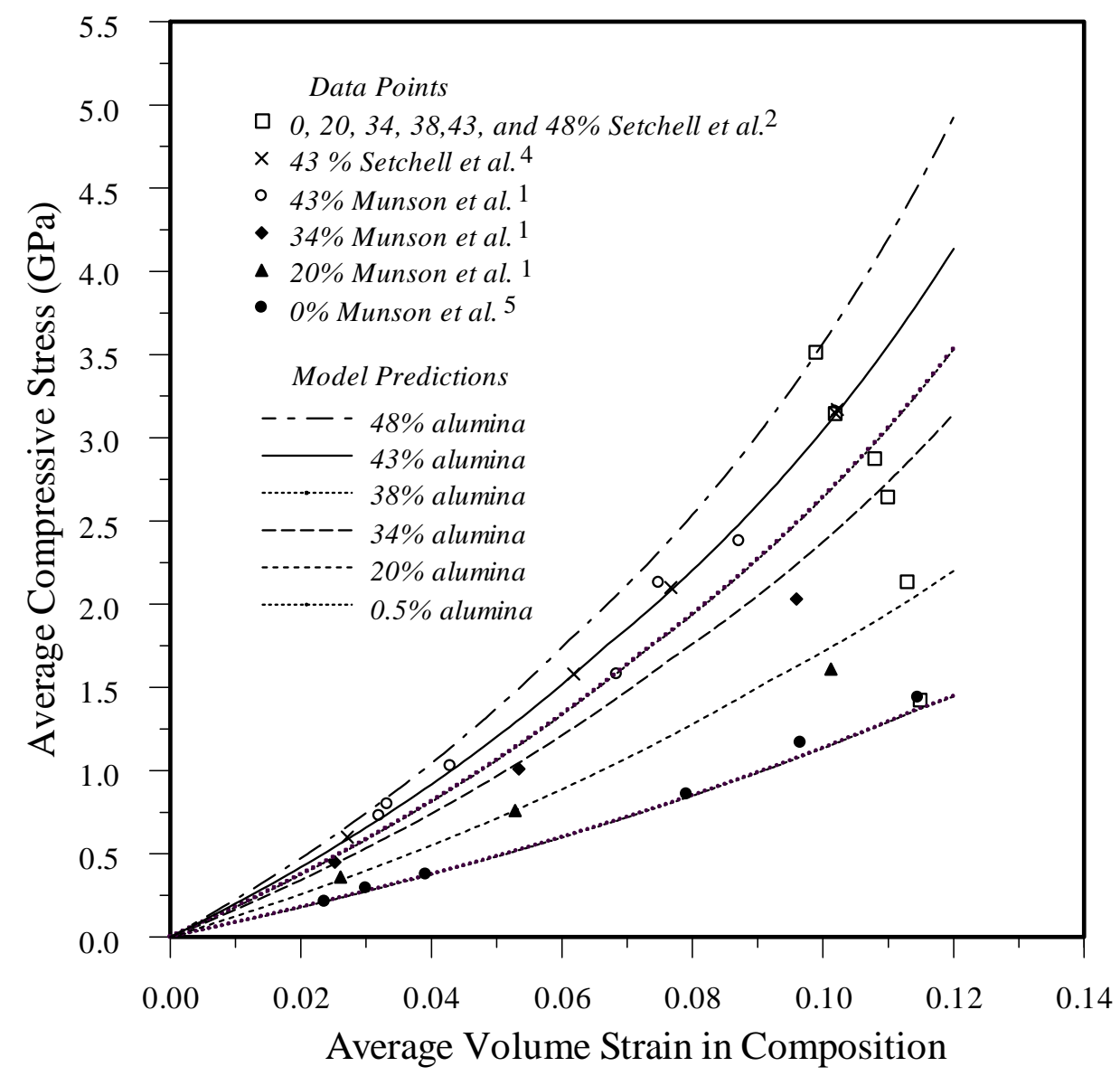

Figure 12. Comparisons of the predicted average normal stress with measurements for the large plane-strain compression for several alumina-filled compositions.

equilibrium Hugoniot stress points for a wide range of compressions have been measured for the composition containing $\sim 43 \%$ alumina by volume. The stresses predicted by the model are in excellent agreement with the stresses determined recently by Setchell et $a$ l. $^{2,4}$ and appear to provide a reasonable match to the stresses determined by Munson et al. ${ }^{1}$ more than thirty years ago. Measurements ${ }^{1}$ of equilibrium stresses for compositions containing 20\% and 34\% alumina are shown in Figure 12 using filled diamonds and triangles. Again it appears that the model predictions are in reasonable agreement with the measurements. It appears that the model stresses are higher than the measurements at compressions above 0.05 . This discrepancy may be due to slight differences between the alumina particles used in those experiments and the alumina particles characterized in this study. The model predictions and the more recent data ${ }^{2}$ show better agreement for the single test condition examined for each composition. A prediction 
is also shown for a composition containing a trace amount of alumina. This curve is compared to data obtained on the unfilled epoxy. ${ }^{5}$ The stresses predicted by the model, while slightly lower, are close to the measurements in this limit of model applicability.

The value of $S$ used in the model predictions was selected to provide a good match between stresses predicted by the model and measured values for the composition with an initial alumina volume fraction of 43\%. Munson and May ${ }^{5}$ conducted plate impact experiments on the unfilled epoxy and fit the equilibrium Hugoniot states for the epoxy to the Mie-Grüneisen equation of state using $S=1.66$. Drumheller ${ }^{3}$ set $S=2$ in a response model he developed for the composition having an initial alumina volume fraction of $43 \%$. It appears that it may be possible to improve the match between predicted and measured equilibrium stresses by adjusting the parameters appearing in the model. However the agreement obtained with the simple model developed above is remarkable. 
This page intentionally left blank. 


\section{Summary and Discussion}

Mechanical response models for alumina-filled epoxy have been developed and characterized for the composite material formed by suspending alumina particles in epoxy. Response models were developed for small linear elastic deformations as well as large hydrostatic and plane-strain compressions. The key results of this study were model formulations general enough to be used across a range of compositions defined by the initial alumina volume fraction and the remarkable agreement found when matching equilibrium Hugoniot stresses measured in plate impact experiments with predictions of the large plane-strain compression model.

A general linear elastic response model was developed for an isotropic composite consisting of uniformly distributed alumina particles bonded perfectly in an epoxy matrix. As a result of characterizing eleven alumina-filled epoxy compositions, it was determined that the linear elastic response for compositions formed by suspending various volume fractions of a single type of alumina particle in epoxy could be specified using the initial volume fraction of the alumina and six model parameters. Four of these model parameters are known elastic properties of the alumina and epoxy used to fabricate the composite. The other two model parameters, called strain concentration coefficients, reflect the interactions between the alumina particles and epoxy. It was shown that these two additional model parameters could be readily determined for a particular composition. Consequently, characterization of the linear elastic response model is no more difficult than characterizing any linear elastic material provided properties of the alumina and epoxy are known.

Analysis of the dependence of the strain concentration coefficients on the initial alumina volume fraction, for the eleven compositions characterized, indicates that the two strain concentration coefficients could be written in forms having a particularly simple dependence on the volume fraction. Consequently, the linear elastic response model could be fully described by the initial alumina volume fraction, two elastic moduli each for the alumina and epoxy, and two fixed strain concentration parameters that described the dependence of the strain concentration coefficients on the initial alumina volume fraction. This result has important implications in characterizing alumina-filled epoxy compositions formed using different alumina particle and epoxy types, and modeling the linear elastic response for compositions having known variations in the initial alumina volume concentration. In the first case, if the elastic properties of the alumina and epoxy are reasonably well known, measurement of the effective bulk and shear 
moduli for a particular composition will provide the strain concentration parameters needed to completely specify a linear elastic response model across a range of compositions having a variation in initial alumina volume fraction. In the second case, because the linear elastic response model depends explicitly on the alumina volume fraction, situations in which there is variation in the initial alumina volume fraction, due to processing uncertainty or alumina settling, a framework exists to allow for the analysis of these situations with a higher level of confidence.

An analysis of the effect of alumina particle morphology and size on the strain concentration parameters indicated only slight differences in the elastic response due to these compositional properties. Since no significant difference in the strain concentration parameters was found for the T64, AA5, and AA18 alumina particles examined in this study, it seems reasonable to conclude effects of particle morphology and size on the equilibrium elastic response are much less significant than the effect of initial alumina volume fraction. Additional studies would be needed to determine the effect of varying the epoxy type, using other particle morphologies, and particle sizes on the linear elastic response model, and the results described above provide a useful foundation for planning additional studies on alumina-filled epoxy or related composites.

As an example of model use, the effect of initial alumina volume fraction on the apparent bulk and shear moduli of the T64 alumina-filled epoxy was calculated. Predictions using the parameters determined from material characterization and from various analytic estimations were compared. It is clear that the large difference in stiffness between the alumina and epoxy limited the usefulness of analytic estimation to very small values of the initial alumina volume fraction. Consequently, estimates of the apparent bulk and shear moduli across a range of possible initial alumina volume fraction would be much improved if the strain concentration parameters could be determined for at least one particular composition of interest.

The extension of the response model to large compression proved to be quite successful in the case of large plane-strain compression as demonstrated by the remarkable agreement between model predictions and measurements of the equilibrium Hugoniot stresses from plate impact experiments. Key to the success of this effort were the observations that, due to the large differences in stiffness between the alumina and epoxy, the alumina volume fraction in the composite would change with compression and the suspension of alumina in the epoxy would respond in a fashion similar to the matrix material of a porous solid. These observations allowed the large compression response model to be developed without explicit consideration of specific 
relations between average strain for the composite and the components of the composite. Any extension of the model to more general large compression strain states would likely need to generalize the model presented in this study.

The analysis of hydrostatic compression indicated that the nonlinear response of epoxy under large compression was sufficient to account for most of the nonlinearity in the compression of the composite. The increase in the volume fraction of the alumina was however still apparent.

The success of the large plane-strain compression response model in matching the equilibrium Hugoniot stresses across a wide range of compositions is remarkable because only the parameter defining the nonlinearity of the epoxy $(S)$ was selected to improve the match between the predictions and measurements. The other parameters appearing in the response model were reasonably well established for the alumina and epoxy or specified by requiring that the large compression model produced the same apparent bulk and shear moduli for a composition as given by the linear elastic response model. The fact that the value for $S$ selected was not significantly different than that measured in plate impact experiments on the unfilled epoxy provides additional credibility to the model.

It should be remarked that, even though the large compression response model performed well in predicting the equilibrium Hugoniot stresses, additional development is required before the model would be able to adequately predict the evolution of non-steady stress waves. Previous studies $^{1,2,4}$ have established that wave propagation through alumina-filled epoxy is dispersive. In fact, the study ${ }^{2}$ on compositional effects on shock-compression of alumina-filled epoxy demonstrated effects due to particle morphology and size as the dispersive wave-front transitioned into a steady wave. The largest effect on the structure of the wave-front was seen to be due to the initial volume fraction of alumina but there were discernable differences due to particle morphology and size. It seems likely that the particle morphology and size effects observed were due to the small size of the wave-front resulting in significant strain gradients in a representative volume of material.

Previous response models ${ }^{1,3}$ have accounted for dispersion by allowing the stress to evolve from an instantaneous value to an equilibrium value in response to the compressive strain. Munson et al. ${ }^{1}$ used a Maxwell response model having different relaxation times when the material was compressing or unloading. Drumheller ${ }^{3}$ presented a model using a single relaxation time for the epoxy but constrained the densities of the composite and alumina to change the 
material response on unloading. It seems reasonable that future development work on the response model described above could accommodate features resulting in wave dispersion by allowing the alumina distention to evolve using a Maxwell type model with the equilibrium value being defined as described above. While unloading of the composite has not been examined in detail here, it is clear that the model described will respond differently on unloading from highly compressed states since the epoxy will unload elastically with an apparent stiffness controlled by the level of compression. 


\section{References}

1. D. E. Munson, R. R. Boade, and K. W. Schuler, J. Appl. Phys. 49, 4797 (1978).

2. R. E. Setchell, M. U. Anderson, and S. T. Montgomery, J. Appl. Phys. 101, 083527 (2007).

3. D. S. Drumheller, J. Appl. Phys. 53, 957 (1982).

4. R. E. Setchell and M. U. Anderson, J. Appl. Phys. 97, 083518 (2005).

5. D. E. Munson and May, J. Appl. Phys. 43, 962 (1972).

6. R. Hill, J. Mech. Phys. Solids 11, 357 (1963).

7. A. J. M. Spencer, Continuum Mechanics (Longman, London and New York, 1980).

8. Y. Benveniste, Mech. Mat. 6, 147 (1987).

9. J. D. Eshelby, Proc. Roy. Soc. Lond. A 241, 376 (1957).

10. J. M. Dewey, J. Appl. Phys. 18, 578 (1947).

11. A product of Resolution Performance Products.

12. Previously available from Shell Chemical Company.

13. T-64 tabular alumina ( 325 mesh) is a product of Alcoa Industrial Chemicals Division.

14. AA5 and AA18 are products of Sumitomo Chemical Company.

15. M. M. Carroll and A. C. Holt, J. Appl. Phys. 43, 1626 (1972). 


\section{Distribution}

\begin{tabular}{|c|c|c|}
\hline 1 & MS 0346 & R. S. Chambers, 1524 \\
\hline & MS 0346 & J. V. Cox, 1524 \\
\hline & MS 0346 & M. A. Neidigk, 1526 \\
\hline & MS 0346 & E. D. Reedy, 1526 \\
\hline & MS 0372 & H. E. Fang, 1524 \\
\hline 3 & MS 0372 & S. T. Montgomery, 1524 \\
\hline & MS 0372 & W. M. Scherzinger, 1524 \\
\hline & MS 0372 & A. S. Gullerud, 1525 \\
\hline & MS 0372 & J. M. Redmond, 1525 \\
\hline 1 & MS 0481 & S. G. Barnhart, 2132 \\
\hline 1 & MS 0847 & P. J. Wilson, 1520 \\
\hline 1 & MS 0862 & B. J. Wells, 2732 \\
\hline 1 & MS 0862 & T. A. Ordenez, 2732 \\
\hline 1 & MS 0871 & R. A. Damerow, 2730 \\
\hline 1 & MS 0878 & M. O. Eatough, 2735 \\
\hline 1 & MS 0878 & R. M. Ferrizz, 2735 \\
\hline 1 & MS 0878 & T. W. Scofield, 2735 \\
\hline 1 & MS 1133 & M. U. Anderson, 5916 \\
\hline 1 & MS 1159 & S. C. Jones, 1343 \\
\hline 1 & MS 1195 & C. S. Alexander, 1646 \\
\hline & MS 1195 & J. L. Wise, 1646 \\
\hline & MS 1322 & J. Robbins, 1435 \\
\hline 1 & MS 1452 & D. L. Damm, 2552 \\
\hline 1 & MS 1452 & A. L. Garcia, 2552 \\
\hline & MS 9042 & T. J. Vogler, 8246 \\
\hline 1 & MS 9404 & B. Song, 8365 \\
\hline 1 & MS 0899 & Technical Library, 9536 (electronic copy) \\
\hline
\end{tabular}

